\title{
Backreaction in Cosmology
}

\author{
S. Schander ${ }^{1,2}$ and T. Thiemann ${ }^{2 *}$ \\ ${ }^{1}$ Perimeter Institute, Waterloo, ON, Canada, ${ }^{2}$ Institute for Quantum Gravity, FAU Erlangen - Nürnberg, Erlangen, Germany
}

In this review, we investigate the question of backreaction in different approaches to cosmological perturbation theory, and with a special focus on quantum theoretical aspects. By backreaction we refer here to the effects of matter field or cosmological inhomogeneities on the homogeneous dynamical background degrees of freedom of cosmology. We begin with an overview of classical cosmological backreaction which is ideally suited for physical situations in the late time Universe. We then proceed backwards in time, considering semiclassical approaches such as semiclassical or stochastic (semiclassical) gravity which take quantum effects of the perturbations into account. Finally, we review approaches to backreaction in quantum cosmology that should apply to the very early Universe where classical and semiclassical approximations break down. The main focus is on a recently proposed implementation of backreaction in quantum cosmology using a Born-Oppenheimer inspired method.

Keywords: quantum gravity, cosmology, space adiabatic perturbation theory, quantum fields in curved spacetimes, backreaction

\section{OPEN ACCESS}

Edited by:

Francesca Vidotto,

Western University, Canada

Reviewed by:

Tomi Sebastian Koivisto,

University of Oslo, Norway

Kazuharu Bamba

Fukushima University, Japan

*Correspondence:

T. Thiemann

thomas.thiemann@fau.de

Specialty section: This article was submitted to

Cosmology,

a section of the journal

Frontiers in Astronomy and Space

Sciences

Received: 07 April 2021 Accepted: 14 June 2021

Published: 23 July 2021

Citation:

Schander S and Thiemann T (2021)

Backreaction in Cosmology.

Front. Astron. Space Sci. 8:692198.

doi: 10.3389/fspas.2021.692198

\section{INTRODUCTION}

The $\Lambda$ cold dark matter $(\Lambda \mathrm{CDM})$ concordance model (Cervantes-Cota and Smoot, 2011; Deruelle and Uzan, 2018; Dodelson and Schmidt, 2021), based on the pillars of the Standard Model of particle physics and general relativity, has shaped our current view of the Universe, and has been the driving force behind many of the breakthroughs of modern cosmology, for example the prediction and the discovery of the cosmic microwave background radiation (Aghanim et al., 2019, 2020; Alpher and Herman, 1948a,b; Gamov 1948a,b; Penzias and Wilson 1965). Modeled by only six parameters (Spergel 2015; Aghanim et al., 2020), it features an impressive simplicity while correctly predicting and fitting most of the cosmological data (Aghanim et al., 2019, 2020). One of the most important assumptions within the $\Lambda \mathrm{CDM}$ paradigm is that the Universe is almost spatially homogeneous and isotropic, especially during its earliest phases, but even today when considered on its largest scales. The resulting simplification of Einstein's equations is remarkable as it reduces the ten coupled non-linear partial differential equations in four variables to two ordinary equations in one variable, with solutions known as the Friedmann-Lemaître-Robertson-Walker (FLRW) solutions (Friedman 1922, 1924; Lemaître 1931; Robertson 1933; Walker 1937).

Obviously, a look at the night sky reveals that the Universe is not homogeneous and isotropic, but is characterized by clusters of galaxies and stars, and large voids inbetween (Blumenthal et al., 1984; Cole et al., 2005; Colless et al., 2001; Ross et al., 2020; Zel'dovich et al., 1982). For explanation, the concordance model assumes that smallest quantum fluctuations of the primordial matter and geometry have been stretched to the present time, thereby generating the observable large scale structure. Importantly, these inhomogeneities on any scale smaller than the observable Universe are presumed to evolve following the underlying FLRW background structure, but conversely their evolution does not affect the global FLRW evolution. More precisely, it is assumed that effects from 
the small scale inhomogeneities onto the largest scales can be neglegted, i.e., there is no substantial backreaction.

Doubts regarding the simplistic nature and the question of backreaction have gained momentum in recent years. In fact, the $\Lambda \mathrm{CDM}$ model, as appealing it may be, leads to the conclusion that approximately $69 \%$ of the energy budget of our Universe consists of a yet unknown fluid, dubbed "dark energy," (Aghanim et al., 2020), and which drives the very recent accelerated expansion of the Universe (Riess et al., 1998; Perlmutter et al., 1999; Peebles and Ratra 2003). Most of the remaining $31 \%$ of the energy budget is credited to another yet unknown form of cold "dark" matter (Peebles 1982; Blumenthal et al., 1984; Aghanim et al., 2020), which provides an explanation for the characteristic rotation and motion of the remaining $6 \%$ of ordinary matter in the Universe. In summary, we are faced with the problem that we are literally in the dark about $94 \%$ of the energy and matter content of the observable Universe.

In recent years, these conceptual problems have been accompanied by important tensions in the estimates of certain cosmological parameters as made by different collaborations (Di Valentino et al., 2020a,b; Pesce et al., 2020). The evaluation of the Hubble constant $H_{0}$ as performed by the Planck collaboration (explicitely assuming a $\Lambda \mathrm{CDM}$ model) gives a value of $H_{0}=$ $(67.27 \pm 0.60) \mathrm{km} /(\mathrm{s} \cdot \mathrm{Mpc})$ (Aghanim et al., 2020), while the SH0ES collaboration finds $H_{0}=(74.03 \pm 1.42) \mathrm{km} /(\mathrm{s} \cdot \mathrm{Mpc})$ (Riess et al., 2019), which in turn is based on the measurements of the Hubble Space Telescope. This leads to a tension at the $4.4 \sigma$ level (Di Valentino et al., 2020a). Furthermore, we point to the (albeit weaker) tensions regarding the measurement of the parameter $S_{8}$, a measure for the matter energy density $\Omega_{m}$ and the amplitude of structure growth $\sigma_{8}$ (Aghanim et al., 2020; Di Valentino et al., 2020b).

On the other hand, the theoretical modeling of the early and very early Universe turns out to be a difficult undertaking, in particular the faithful consideration of all interactions within coupled quantum cosmological-matter systems. Since classical cosmological perturbation theory and its various applications to the physics of our Universe (Durrer, 2004; Mukhanov, 2005), represents a successful formalism to model (most of) the cosmological data today, one of the most promising approaches to make progress in the field is to consider an inhomogeneous, but perturbative, quantum cosmology, i.e., to establish a quantization of the well-known (possibly) gaugeinvariant cosmological perturbation theories (Brandenberger et al., 1993; Brandenberger, 2004; Elizaga Navascués et al., 2016). In fact, there has been tremendous progress in developing such quantum cosmological perturbation theories, for example, in quantum geometrodynamics (Kiefer, 2007; Brizuela and Krämer, 2018), in string cosmology (Erdmenger, 2009), as well as in loop quantum cosmology (LQC) and spinfoam cosmology (Bianchi et al., 2010; Vidotto 2011; Cailleteau et al., 2012; Agullo et al., 2013; Elizaga Navascués et al., 2016), to mention but a few. Unfortunately, the majority of these approaches neglect backreaction effects from the inhomogeneous quantum fields on the homogeneous, dynamical degrees of freedom, or incorporate a series of assumptions which are hard to control, similar to the situation in classical cosmological perturbation theory.

It seems hence very timely to scrutinize and question the various assumptions of the concordance model of cosmology, and to develop suitable formalisms which are able to take interactions in coupled (quantum) cosmological models more realistically and unambigiously into account. In this review, we start by assessing the question of backreaction, i.e., whether cosmological inhomogeneities have an effect on the large scale evolution of the Universe, especially in view of the occurent inconsistencies within the standard model. We consider different aspects of backreaction, in particular we discuss backreaction in classical, semiclassical and quantum mechanical models. Our main focus is on the purely quantum mechanical backreaction and we discuss one recent approach to including backreaction in quantum cosmology in more detail (Schander and Thiemann, 2019a). The structure of the paper is then as follows.

In Section 2, we provide an overview of the results in the field of classical backreaction, which is particularly relevant for late time cosmological models. In Section 3, we consider semiclassical backreaction which occurs when considering quantum fields on classical curved space times. Section 4 gives an overview of quantum backreaction, i.e., backreaction that occurs in purely quantum theoretical models. In Section 5, we focus on one particular approach to quantum backreaction which uses mathematical tools inspired by the Born-Oppenheimer approximation. Section 6 provides a final discussion and an outlook.

\section{CLASSICAL BACKREACTION}

Standard perturbative approaches to cosmological perturbation theory implicitely conjecture that backreaction, i.e., the effects of cosmological inhomogeneities on the global or macroscopic evolution of the Universe can be ignored. For purely classical models of the Universe that are particularly relevant for its late time evolution, this conjecture has generated an intense debate over the last decades. And still, there is no consensus on the question of backreaction in the classical regime, see for example the reviews by Clarkson et al. (2011), Ellis (2011), and Bolejko and Korzynski (2017).

The question of backreaction is closely related to the fitting problem, (Ellis and Stoeger, 1987), and the problem of averaging, (Clarkson et al., 2011). In fact, an intuitive way to access the effects of small inhomogeneities on the macroscopic scales is to construe an averaging procedure that defines new homogeneous variables by integrating the inhomogeneous fields over a certain space time domain, and to compare their properties and dynamics to the assumed FLRW Universe, (Ellis, 2011). However, it is inadmissible to conclude from the validity of Einstein's equations for the inhomogeneous fields on the smallest scales (where they have been excellently checked), that the averaged fields satisfy the Einstein equations (Paranjape, 2012). This is because evaluating the Einstein tensor and taking a space (time) average does not commute in general. Hence, the averaging procedure can lead to additional 
contributions to Einstein's equations that might be considered as effective source terms for the geometry, see for example (Buchert, 2000, 2001).

As it turns out, the results regarding the form and strength of backreaction depend heavily on the averaging procedure and the matter model being chosen, as well as on the choice of space time volumes to be integrated over. In the non-perturbative regime, the two most discussed averaging procedures are the scalar averaging scheme by Buchert (2008) and the Macroscopic Gravity approach by Zalaletdinov (1997, 2008). Buchert's scheme focuses on building spatial averages of scalar fields and derives effective (scalar) equations of motion for the averaged quantities, for example an improved Raychaudhuri equation for the averaged scale factor that includes a kinematical backreaction term. While being technically easy to implement, the Buchert scheme relies on a system of scalar equations that is not closed (Clarkson et al., 2011), and consequently requires additional information to fix the solution. Besides, the averaging demands to fix suitable spatial domains and hence, a hypersurface slicing. In contrast, the Macroscopic Gravity approach is manifestly covariant but requires to define an auxiliary so-called bi-local transport operator, (Ellis, 2011). Physical applications of these schemes yield a range of different results, ranging from explaining the recent accelerated expansion of the Universe or the $H_{0}$-tension (Buchert and Räsänen, 2012; Heinesen and Buchert, 2020), to negligible backreaction effects (Paranjape and Singh, 2007, 2008).

Many of the afore-mentioned approaches (among many others) assume the matter content to be modeled by a fluid, which is likely to be a poor approximation to the true lumpy late time Universe. Models with more realistic matter distributions are for example the Timescape Cosmology by Wiltshire (2009), who separates the Universe into underdense expanding regions bounded by overdense virialized structures, the Swiss Cheese Model (Kantowski, 1969; Tomita, 2000; Biswas and Notari, 2008), or modifications of FLRW Universes that cut spherically symmetric Lemaître-Tolman-Bondi or Szekeres dust space time regions (Marra et al., 2008; Bolejko and Celerier, 2010), to mention but a few. By construction, many of these models follow the evolution of an appropriately fitted FLRW model since they assume a background structure from the beginning. Consequently, they do not attack the backreaction problem outlined before. In contrast, the model by Lindquist and Wheeler (1957) assembles static Schwarzschild regions without relying on any background, and which has been further investigated by Clifton (2011) and Clifton and Ferreira (2009). In both cases, the models provide insights into backreaction effects on light propagation (Krasinski and Bolejko, 2011; Sussman, 2011), which points to another important topic.

In fact, cosmological observations such as the distance-redshift relation or the angular diameter distance rely on measurements of light, traveling along our past lightcone in a very inhomogeneous Universe. The seminal work by Kristian and Sachs (1966) laid out the basis for analyzing backreaction on light propagation. Flanagan (2005), for example, used these ideas to compute the deceleration parameter as measured by comoving observers. Gasperini et al. (2011) define a covariant light-cone average for the backreaction problem, see also (Fanizza et al., 2020) for a more recent generalized proposal. Räsänen (2009) and Räsänen (2010) derives a relationship of the redshift and the angular diameter distance to the average expansion rate for statistically homogeneous and isotropic universes, based on Buchert's approach, and Barausse et al. (2005) and Bonvin et al. (2006) evaluate the distance-redshift relation and the luminosity distance in a perturbative framework. Most recently, Heinesen (2021a), Heinesen (2021b) and Koksbang (2019), Koksbang (2020), Koksbang (2021) investigated the effects of inhomogeneities and averaging on a possible redshift drift.

Many approaches that attempt to make direct contact with cosmological observations restrict their analysis to cosmological perturbation theory in an FLRW Universe, as opposed to the above-mentioned non-perturbative approaches. Most of them consider flat $\Lambda \mathrm{CDM}$ models with Gaussian scalar perturbations as initital conditions. To evaluate backreaction, they compute the deviations to the Hubble expansion rate or similar variables that are caused by backreaction (Brandenberger et al., 2018; Clarkson et al., 2009; Kolb et al., 2010; Kolb et al., 2005; Li and Schwarz, 2008), or give effective Friedmann equations with additional contributions (Paranjape and Singh, 2007; Behrend et al., 2008; Brown et al., 2009; Peebles, 2010; Baumann et al., 2012). The idea is to perform appropriate spatial averages of the perturbed quantities and to use the given statistical information of the perturbation fields in guise of their power spectra. It turns out that due to the smallness of the gravitational potential and the power suppression of modes on large scales, backreaction for the expansion rate is always small. However, the backreaction to the deceleration parameter $q$ and the variance of the Hubble rate depend on an auxiliary UV-cutoff that might lead to large backreaction even if it is set by scales larger than the non-linearity scale (Clarkson et al., 2011). Other approaches to backreaction in the linear regime are (Baumann et al., 2012) who propose a reformulation of perturbation theory that leads to small backreaction on the largest scales but affects the baryon accoustic oscillations, and (Green and Wald, 2011, 2012, 2013, 2014) who claim, using a point limit process, that backreaction can never mimic dark energy and put strong constraints on its strength.

We also point to the quite recent advent of numerical tools that allow to simulate increasingly realistic models of the Universe, including relativistic effects (Löffler et al., 2012; Mertens et al., 2016) and N-body simulations (Adamek et al., 2016; Barrera-Hinojosa and Li, 2020). Using the N-body relativistic code "gevolution," Adamek et al. (2019) find that backreaction on the expansion rate in a $\Lambda \mathrm{CDM}$ and an Einstein-de Sitter Universe remains small if one chooses averaging volumes related to the Poisson gauge, while when choosing comoving gauge backreaction is of the order of $15 \%$. Other works in this respect were done by Macpherson et al. (2019), who also claim that backreaction effects are small, however based on a fluid approximation which breaks down as soon as it comes to shell crossing.

Finally, let us also point to the consideration of backreaction from long wavelength modes of the early Universe. In this respect, early contributions were notably made by Tsamis and Woodard 
(1993, 1996), as well as by Abramo et al. (1997) and Mukhanov et al. (1997). The latter works pursue a gauge-invariant formulation of the backreaction problem associated with an effective long wavelength energy momentum tensor, and within a slow-roll inflationary scenario. Unruh (1998) subsequently examined the question of whether this effect is indeed locally measurable, and it was found that such backreaction effects (in single field inflationary theories) can be absorbed by a gauge transformation, (Abramo and Woodard, 2002; Geshnizjani and Brandenberger, 2002). However, backreaction of such fluctuations becomes locally measurable after introducing an additional (subdominant) clock feld, (Geshnizjani and Brandenberger, 2005). This approach was extended by Marozzi et al. (2013) based on the gauge-invariant formalism by Finelli et al. (2011), and secondly by Brandenberger et al. (2018) beyond perturbation theory. Further contributions were made by Losic and Unruh (2005), Losic and Unruh (2008) who support the idea that backreaction represents a real and measurable effect.

\section{SEMICLASSICAL BACKREACTION}

For considerations of backreaction in models of the very early Universe, the standard model of cosmology suggests that (at least) the matter fields should be studied in a quantum mechanical framework. The implementation of such ideas can be realized via different paths, and we consider here the approaches of semiclassical gravity (Ford 2005; Wald 1977, 1978) and stochastic (semiclassical) gravity (Calzetta and $\mathrm{Hu}$ 1987; Hu and Verdaguer 2008; Jordan 1986, 1987). Both approaches rely on the framework of quantum field theory on curved space times (QFT on CST) (Birrell and Davies, 1984; Fulling 1989), which itself takes the effects of the classical curved space times on the quantum matter fields into account but in general not the backreaction effects of those quantum fields on the classical background.

The backreaction problem in semiclassical gravity was first brought in by Wald (1977) who considered the backreaction from particle creaction on a gravitational field. The idea of the semiclassical program is to consistently define an improved set of Einstein field equations in which the expectation value of the quantum stress-energy tensor $\boldsymbol{T}_{\mu \nu}$ of the matter fields with respect to an appropriate quantum state of the matter fields $\omega$ appears as a source term,

$$
R_{\mu \nu}+\frac{1}{2} g_{\mu \nu} R=8 \pi G \omega\left(: T_{\mu \nu}:\right)
$$

where the quantization with respect to the matter fields is expressed using bold letters and the dots indicate the normal ordering of the stress-energy tensor. The state $\omega$ should be considered as a positive linear functional in the sense of the algebraic approach to quantum field theory (QFT) (Haag 1992; Araki 1999).

The first goal of semiclassical gravity is to define a procedure that leads to a meaningful expression for the expectation value of the stress-energy tensor. In fact, the latter depends on products of operator-valued distributions, even for the simple case of a real-valued Klein-Gordon field, and its expectation value is in general a divergent expression. Wald (1977) gave a set of axioms that are required to hold for a suitable renormalization scheme. Possible proposals are the Hadamard point-splitting method (Brunetti and Fredenhagen, 2000; Hollands and Wald, 2001), and the adiabatic regularization procedure (Fulling and Parker 1974; Fulling et al., 1974; Parker and Fulling, 1974). In either scheme, the result of the regularization procedure is a set of modified "semiclassical" Einstein equations. These equations are substantially harder to solve than the original Einstein equations and many studies restrict to cases of conformally coupled matter to avoid problems regarding the well-posedness and the stability of the solutions (Ford, 2005). Caution is also required regarding the question of self-consistency of the backreaction effects, as has been discussed by Flanagan and Wald (1996).

Many applications of the semiclassical gravity approach to early Universe cosmology have been considered. For example, Fischetti et al. (1979) analyzed the backreaction effects from a conformally invariant matter field in an FLRW Universe with classical radiation, and found that the trace anomaly can soften the cosmological singularity, but not avoid it. Other works in this direction were done by Anderson (1983, 1984, 1985), who also considered the trace effects on the particle horizon. A well-known example of trace anomaly effects from semiclassical gravity is the Starobinsky (1987) cosmological model, which has however not survived the observational scrutiny of the Planck data (Ade et al., 2016).

Another application of semiclassical gravity is the study of backreaction of particle creation on the dynamics of the early Universe, as already conceived by Wald (1977). Grishchuk (1977) as well as Hu and Parker (1977) considered the effect of gravitons around the Planck time in an FLRW Universe with a classical, isotropic fluid. The model leads to a timely non-local (i.e., history-dependent) backreaction effect, ( $\mathrm{Hu}$ and Verdaguer, 2020). Similar studies were performed for anisotropic FLRW Universes and it was shown that particle production due to the shear anisotropy isotropizes space time (Hu and Parker, 1978; Zel'dovich and Starobinsky, 1972). Regarding the effects of particle creation in a spatially inhomogeneous but isotropic Universe, we refer to the work by Campos and Verdaguer (1994).

We also point to the more recent works by Finelli et al. (2002), Finelli et al. (2004) who specifically consider a slow-roll (almost de Sitter) phase of the very early Universe and compute a(n adiabatically) renormalized energy momentum tensor of quantum inflaton, respectively cosmological scalar fluctuations. In case of the cosmological scalar perturbations, they found that the energy momentum tensor is characterized by a negative energy density which grows during inflation, and also that backreaction is not a mere gauge artifact.

Further contributions to the topic of semiclassical gravity for cosmological situations were notably made by Dappiaggi et al. (2008); Dappiaggi et al. (2010); Eltzner and Gottschalk (2011); Gottschalk and Siemssen (2018); Hack (2013); Matsui and 
Watamura (2020); Parker and Raval (1999); Pinamonti (2011), to mention but a few. Most recently, Meda et al. (2020) and Pinamonti and Siemssen (2015) have made progress on the definition of the semiclassical theory for general couplings by proving existence and uniqueness of solutions in flat cosmological space times with a massive quantum scalar field. The idea of relating backreaction effects to the decay of a cosmological constant has for example been promoted by Dymnikova and Khlopov (2001). We also point to the work by Matsui and Watamura (2020) (and references therein) who claim that the approach of semiclassical gravity is not appropriate to describe the early Universe.

The second approach to evaluating backreaction in semiclassical cosmology that we present here, denoted stochastic gravity (Hu and Verdaguer 2020), creates a link to open system concepts and statistical features such as dissipation, fluctuations, noise and decoherence. It employs a so-called closed time path coarse grained effective action (CTP CGEA) (Calzetta and Hu 1987; Jordan 1986, 1987), in order to derive a set of modified semiclassical Einstein equations, denoted as Einstein-Langevin equations. It includes the semiclassical approach but extends it by a stochastic noise term ( $\mathrm{Hu}$ and Matacz, 1995).

Some of the first applications of the CTP CGEA formalism to the backreaction problem in cosmology were made by Calzetta and $\mathrm{Hu}$ (1987, 1989, 1994). Hu and Matacz (1995) derived the Einstein-Langevin equations for the case of a free massive scalar field in a flat FLRW background, as well as for a Bianchi Type-I Universe. The case of a massless conformally coupled field was discussed in (Campos and Verdaguer, 1994). The scope of works includes topics such as stochastic inflation, where quantum fluctuations present in the noise term backreact on the inflaton field (Calzetta and $\mathrm{Hu}, 1995$; Lombardo and Mazzitelli, 1996), as well as studies of the reheating phase in inflationary cosmology (Boyanovsky et al., 1995; Ramsey and Hu, 1997). The formalism was also used by Sinha and Hu (1991) to check the validity of the minisuperspace approximation in quantum cosmology. Further applications can be found in the paper and textbook by $\mathrm{Hu}$ and Verdaguer (2008), Hu and Verdaguer (2020).

We also point to one of the most prominent applications of stochastic methods to early Universe cosmology by Starobinsky (1982) and Starobinsky (1988). His stochastic inflationary model evaluates backreaction of small scalar field quantum perturbations on the corresponding long wavelength modes (which are assumed to behave classically) by additional stochastic terms in the long wavelength equations of motion. A slow-roll behavior of the background is assumed. Interestingly, it has been shown that the stochastic and the quantum feld theoretic approaches to perturbations in the early Universe yield the same results, (Starobinsky and Yokoyama, 1994; Finelli et al., 2009; Tsamis and Woodard, 2005). For recent considerations of stochastic inflation beyond the (strict) slow-roll conditions, we refer to the work by Pattison et al. (2019) and references therein.
Both approaches, semiclassical as well as stochastic gravity regard the gravitational field as a classical entity from the start while the matter fields are considered to be of quantum nature. While this represents a seminal progress to incorporating quantum effects of the matter fields in the early Universe, it can and should be questioned whether this somehow incompatible approach (classical and quantum fields treated at the same level) survives the test of future observations, and whether it should be replaced by a more consistent approach quantum gravity - at least for the earliest moments of the cosmic history.

\section{APPROACHES TO QUANTUM BACKREACTION}

The question of backreaction in quantum gravity and quantum cosmology encompasses a variety of different approaches and definitions of backreaction. In quantum cosmology, backreaction is usually identified as the effects from the inhomogeneous quantum perturbation fields on the (quantum) homogeneous and isotropic degrees of freedom, which is also the notion of backreaction used in the next section (Schander and Thiemann, 2019c). This approach is tightly related to a perturbative expansion with respect to the inverse Planck mass $m_{\mathrm{Pl}}^{-1}=(G / \hbar)^{1 / 2}$, where $G$ is Netwon's constant and we set $\hbar \equiv 1$. More precisely, it employs a Born-Oppenheimer type scheme (Born and Oppenheimer, 1927), with respect to $m_{\mathrm{Pl}}^{-1}$. We will thus focus on implementations of the Born-Oppenheimer method to quantum gravity and quantum cosmology.

In fact, the idea that quantum gravity can be considered as a perturbative theory with respect to $m_{\mathrm{Pl}}$ was already introduced by Brout (1987). The first investigations of backreaction in quantum gravity that rely on this expansion were performed in the framework of quantum geometrodynamics (Wheeler, 1957; Kiefer, 2007). The idea is to expand the Wheeler-DeWitt equation in terms of the ratio of the Planck mass and the matter field mass (Kiefer and Singh, 1991). A different idea, conceptually similar to the schemes considered here, is to use a Born-Oppenheimer type approach, relying on the same perturbation parameter. Different considerations of the problem (giving rise to similar results) can be found in the works by Bertoni et al. (1996), Brout and Venturi (1989), and Kiefer (1994) (for a summary, see Kiefer, 2007). A review of the ideas of Born and Oppenheimer will be given in the next section, but to understand its use in the given context we present the key ideas.

For simplicity, let $Q$ denote the gravitational and $q$ the matter degrees of freedom. The Born-Oppenheimer scheme employs an ansatz solution for the quantum Hamiltonian and momentum constraint of the form (Kiefer, 2007),

$$
\Psi(q, Q)=\sum_{n} \chi_{n}(Q) \psi_{n}(q, Q),
$$

where $\left\{\psi_{n}(q, Q)\right\}_{n}$ is supposed to be a known orthonormal basis of the matter Hilbert space that solves the matter part of 
the constraint and $Q$ is to be considered as an external parameter for this eigenvalue problem. Then, one applies the constraints to $\Psi$ and applies some $\psi_{k}(q, Q)$ from the left (i.e., one considers the inner product of the matter states). This gives rise to constraint equations for the geometric factors $\chi_{n}(Q)$, which can be seen as an effective quantum problem for the geometric part, including the backreaction effects of the quantum matter system. In this scenario, the Born-Oppenheimer approximation consists in neglecting the contributions that enter with higher orders in $m_{\mathrm{Pl}}^{-1}$.

In order to extract physical results from the formalism, one can additionally employ a semiclassical approximation (which is however independent of the Born-Oppenheimer approach). This should yield the semiclassical limit of quantum gravity, i.e., a matter QFT on CST. It is common to employ a WKB ansatz for the geometrical states $\chi_{n}(Q)$ of the form,

$$
\chi_{n}(Q)=C_{n}(Q) e^{i m_{\mathrm{Pl}}^{2} S[Q]},
$$

where $S[Q]$ stands for the geometric action in the geometrodynamical approach. The perturbative scheme in $m_{\mathrm{Pl}}^{-1}$ eventually yields the semiclassical Einstein equations. In this sense, these approaches evaluate the backreaction of the quantum matter fields on the quantum or classical geometry.

One can apply the Born-Oppenheimer and WKB approximations in a different manner. Instead of taking the expectation value with respect to the quantum matter system, one applies the Wheeler-DeWitt constraints on the total Born-Oppenheimer ansatz function and uses the WKB approximation for the geometrical part. Restricting again to the lowest order with respect to the inverse Planck mass, this yields a quantum constraint for the matter wave function which depends on the classical action (through the WKB ansatz), and derivatives with respect to the spatial metric thereof. The idea of the above-cited works (and also of Briggs and Rost, 2000) is to introduce an external time parameter that depends on this derivative, hence giving rise to a Schrödinger equation for the matter system that includes the backreaction of the geometry through the geometry-dependent time derivative. In fact, this gives rise to a notion of time in a formerly background independent framework. Such ideas go back to DeWitt (1967) and have been applied to a variety of cosmological situations (see (Kiefer 2007) and references therein). It is however a different notion of backreaction than the one considered in the next section. Besides, the present approach uses a Born-Oppenheimer approach plus a semiclassical WKB approximation while the next section uses the purely quantum mechanical space adiabatic perturbation extension of the Born-Oppenheimer scheme. Applications of the former works to the inflationary paradigm with perturbations and a discussion of the question of unitary evolution of the perturbations can be found in the work by Chataignier and Krämer (2021) and references therein. They also consider cosmological perturbations that include gravitational contributions (i.e., the Mukhanov-Sasaki variables). Similar approaches that do not split the system into geometric and matter parts but include (perturbative) parts of the gravitational degrees of freedom in the fast subsystem and (homogeneous) matter parts in the slow sector were already presented by Halliwell and Hawking (1985) and Vilenkin (1989). This choice is also used in Schander and Thiemann (2019c).

The Born-Oppenheimer approximation was also considered within approaches to quantum gravity with other variable choices. Giesel et al. (2009) aimed at an application of the Born-Oppenheimer methods to loop quantum gravity (LQG) (Thiemann, 2008; Rovelli, 2010), using holonomy-flux variables or connection-flux variables. As it turns out, this choice of variables prevents the use of the Born-Oppenheimer methods since the flux operators are mutually non-commuting (which is a prerequisite for the Born-Oppenheimer scheme). Instead, they use commuting co-triad variables for the gravity sector and a scalar field for the matter sector to derive the semiclassical Einstein equations that take the backreaction of the quantum matter fields via an expectation value into account. Giesel et al. (2009) consider their model on a discrete lattice (as it is common practice for approaches to LQG), and thus formally obtain a lattice QFT on a discrete curved space time. They also point to the possibility of pursuing the formal Born-Oppenheimer scheme and computing quantum solutions to the gravity sector with the effective backreaction of the quantum matter fields. Besides, they introduce a hybrid approach (similar to the models we consider here) where the gravitational sector is restricted to FLRW solutions and the fast part of the system is given by the matter quantum fields. They also propose to introduce coherent states for the gravitational subsystem in order to make progress in finding solutions. Due to the complexity of the gravity-matter systems, the focus of this work lies on spelling out the conceptual ideas rather than technically carrying out the program in detail.

More recently, Stottmeister and Thiemann (2016a,b,c) considered similar questions in the context of LQG but employed the more general scheme of space adiabatic perturbation theory (SAPT) (Panati et al., 2003). Since in the latter approach, the variables of the slow, gravitational sector are not required to commute, it is in principle possible to apply the Born-Oppenheimer ideas also to LQG and related theories. The concrete implementation turns however out to be difficult due to the particular structure of the LQG phase space (which relies on a cotangent bundle of a compact Lie group rather than on a vector space) and its quantum represention. Other open issues of their attempts are related to the underlying graph structure of LQG models and the projective limits of finite dimensional truncations of the gravitational phase space that are needed in order to construct a continuum theory (Stottmeister and Thiemann, 2016c). They also point out that a major obstruction to the derivation of a QFT on CST from LQG lies in the inequivalent representations of quantum fields for different gravitational configurations (Stottmeister and Thiemann, 2016c). This problem is a generic feature of background dependent quantum field theories. In this work, we present a (perturbative) solution to this problem which makes the application of space adiabatic methods to quantum cosmology possible (Fernandez-Mendez et al., 2012; Castelló Gomar et al., 2015; Castelló Gomar et al., 2016). 
For completeness, we also mention the application of Born-Oppenheimer methods within the spinfoam approach to LQC (Rovelli and Vidotto, 2008) (see Ashtekar et al., 2003; Bojowald, 2008 for the LQC approach), and that Castelló Gomar et al. (2016) consider a conceptually different kind of Born-Oppenheimer approximation in the hybrid approach to LQC. These seminal works make important progress by first considering the problem of backreaction, but must either remain on a rather formal level or include various assumptions which are hard to control. We therefore advocate to employ the SAPT scheme presented in the next section which serves as an unambigious, rigorous and perturbative approach, in principle applicable to any quantum cosmological framework and realizable up to any perturbative order, to taking quantum cosmological backreactions thoroughly into account. The approach applies to a much wider variety of quantum systems in comparison to the Born-Oppenheimer approach (in particular to quantum cosmological perturbation theory), does not rely on the introduction of semiclassical ansatz states and iteratively provides quantum constraints or equations of motion whose solutions approximate the true solutions up to, in principle, indefinitely small errors.

\section{QUANTUM BACKREACTION WITH SPACE ADIABATIC METHODS}

Computing and including backreaction in (perturbative) quantum cosmology requires an approximation scheme that ideally takes the physical characteristics of the system into account. SAPT as proposed by Panati et al. (2003) and extensions thereof are ideally suited to achieve this goal and to integrate backreaction effects into quantum cosmology (Schander and Thiemann, 2019a; Schander and Thiemann 2019b; Schander and Thiemann 2019c; Neuser et al., 2019).

SAPT is a generalization of the well-known Born-Oppenheimer approximation for non-relativistic molecular systems. Both approaches exploit the small ratio of two internal parameters such as the mass ratio of electrons and nuclei in a molecule to define a perturbation parameter,

$$
\varepsilon^{2}:=\frac{m_{\mathrm{e}}}{m_{\mathrm{n}}} \approx 5.46 \times 10^{-4} \ll 1,
$$

with the electron mass $m_{\mathrm{e}} \approx 9.11 \times 10^{-31} \mathrm{~kg}$ and the nuclei mass $m_{\mathrm{n}} \approx 1.67 \times 10^{-27} \mathrm{~kg}$. In the simplest atom with one nucleus and one electron (although exact solutions are known for this case), the Hamilton function has the form,

$$
H(q, P ; x, y)=\frac{\varepsilon^{2} P^{2}}{2 m_{\mathrm{e}}}+\frac{y^{2}}{2 m_{\mathrm{e}}}+V(q ; x),
$$

where $(q, P)$ and $(x, y)$ are the canonically conjugate pairs of the nucleus and the electron respectively. $V(q ; x)$ is a smooth potential, typically a Coulomb potential depending on the distance between nucleus and electron. In this molecular set up, the equipartition theorem states that the kinetic energies of nuclei and electrons are of the same order, and hence, on average, the nuclei move much slower than the electrons with correspondent statistically-averaged velocities, $\left\langle v_{\mathrm{n}}\right\rangle \approx \varepsilon\left\langle v_{\mathrm{e}}\right\rangle$.
Born and Oppenheimer used this fact to define suitable ansatz solutions for the quantum mechanical problem: On the typical electronic time scale, the nuclei are at rest and the non-trivial electronic contributions of the Hamilton operator can be considered at fixed nuclei positions $q \in \mathbb{R}$,

$$
\boldsymbol{H}_{\mathrm{e}}(q):=\frac{\boldsymbol{y}^{2}}{2 m_{\mathrm{e}}}+\boldsymbol{V}(q, \boldsymbol{x}),
$$

where the bold letters $\boldsymbol{y}, \boldsymbol{x}$ denote momentum and position operators of the electron defined on their respectively dense domains in $L^{2}(\mathbb{R}, \mathrm{d} x)$. Ideally, the operator function $\boldsymbol{H}_{\mathrm{e}}(q)$ admits a solvable $q$-dependent eigenvalue problem,

$$
\boldsymbol{H}_{\mathrm{e}}(q) \xi_{n}(q)=E_{n}(q) \xi_{n}(q)
$$

with a discrete $q$-dependent eigenbasis $\left\{\xi_{n}(q)\right\}_{n \in \mathbb{N}}$ in the fast Hilbert space $\mathcal{H}_{\mathrm{f}}:=L^{2}(\mathbb{R}, \mathrm{d} x)$, for which the so-called electronic energy bands $E_{n}(q)$ are gapped functions, i.e., $E_{n}(q)-E_{m}(q) \neq 0$ pointwise for $m \neq n$. One can use this eigenbasis as an ansatz solution for the full Hamilton operator $\widehat{\boldsymbol{H}}$ (the Weyl quantization with respect to the nuclei sector is labeled by hats), and ask whether it provides an approximate solution to the entire problem. Equivalently, we can define for every electronic eigensolution the direct integral operator,

$$
\widehat{\boldsymbol{\pi}}_{n}:=\int_{\mathbb{R}}^{\oplus} \mathrm{d} q \xi_{n}(q)\left\langle\xi_{n}(q), \cdot\right\rangle_{\mathrm{e}}=\int_{\mathbb{R}}^{\oplus} \mathrm{d} q \pi_{n}(q),
$$

on the total Hilbert space $\mathcal{H}=L^{2}(\mathbb{R}, \mathrm{d} q) \otimes \mathcal{H}_{\mathrm{f}}$ and ask whether it commutes with $\widehat{\boldsymbol{H}}$. Of course, the answer is in the negative, but it turns out that the commutator scales like $\varepsilon$,

$$
\left[\widehat{\boldsymbol{H}}, \widehat{\boldsymbol{\pi}}_{n}\right] \sim \mathcal{\varepsilon} .
$$

This is because of the adiabatic relation between the electrons and the nuclei. By construction, the Weyl quantization $\widehat{\boldsymbol{H}}_{\mathrm{e}}(\widehat{q})$ of the electronic Hamiltonian and $\widehat{\boldsymbol{\pi}}_{n}$ commute. However, the remaining contribution to the Hamilton operator $\widehat{\boldsymbol{H}}$, in particular the kinetic energy of the nucleus, scales like $\varepsilon^{2}$ and leads hence to the estimate in Eq. 9. The Born-Oppenheimer approximation builds on this result and proposes to use the ansatz functions,

$$
\Psi(q ; x)=\sum_{n} \psi_{n}(q) \xi_{n}(q ; x),
$$

to solve the full quantum problem. In its simplest version, the scheme neglects any of the contributions that arise from applying the kinetic energy operator of the nucleus to the electronic ansatz functions (as they enter with small $\mathcal{\varepsilon}$-factors), and thus results in an effective eigenvalue problem for the nucleus only,

$$
\left(\frac{\widehat{P}^{2}}{2 m_{\mathrm{n}}}+E_{n}(\widehat{q})\right) \psi_{n}(q) \equiv E \psi_{n}(q)
$$

If this nucleonic eigenproblem can be solved, the scheme leads in fact to viable results for the stationary energy spectra of molecules, which are given by the energy solutions $E$, and 
which include the backreaction of the electrons via the potential energy $E_{n}(\widehat{q})$.

Unfortunately, the Born-Oppenheimer approach comes with some limitations which preclude its application to more complicated systems. Firstly, the scheme explicitely uses that the electronic eigenfunctions depend only on the configuration variable of the nucleus. Was the coupling between electrons and nuclei provided by non-commuting slow operators, for example by $\hat{q}$ and $\hat{p}$, the scheme would fail since the direct integral construction in Eq. 8 builds on the commutativity (i.e., the existence of a common spectrum) of the coupling operators. Also one could not define the ansatz functions in Eq. 10. Secondly, the scheme does not provide a simple extension to better error estimates. This becomes problematic if one is interested in the dynamical evolution of the system. The interesting dynamics of the nuclei happens on time scales $t_{\mathrm{n}} \sim t_{\mathrm{e}} / \varepsilon$ or larger, but considering the evolution generated by $\widehat{\boldsymbol{H}}$ with respect to the above ansatz functions, the scheme cannot lead to trustworthy results due to the commutator relation Eq. 9.

It should be possible to do better. In fact, the adiabatic theorem (Teufel, 2003) states that under certain conditions (to be discussed in the sequel), there exists an orthogonal projection operator $\widehat{\Pi} \in \mathcal{B}(\mathcal{H})$ in the bounded operators on the total Hilbert space $\mathcal{H}$ such that,

$$
[\widehat{\boldsymbol{H}}, \widehat{\boldsymbol{\Pi}}]=\mathcal{O}_{0}\left(\varepsilon^{\infty}\right),
$$

where the right hand side means that for all $m \in \mathbb{N}$, there exists a constant $C_{m}<\infty$ such that $\|[\widehat{\boldsymbol{H}}, \widehat{\Pi}]\|_{\mathcal{B}(\mathcal{H})} \leq C_{m} \varepsilon^{m}$, in the norm of bounded operators on $\mathcal{H}$. Most importantly, $\widehat{\Pi}$ can be constructed by a "semiclassical symbol" function, i.e., an operator-valued ansatz function like the almost-projector function $\pi_{n} \in C^{\infty}\left(\mathbb{R}, \mathcal{B}\left(\mathcal{H}_{\mathrm{f}}\right)\right)$ from the simple example above. This symbol function appears as an asymptotic series in the perturbation parameter $\varepsilon$, and-to anticipate the result-the equivalent of $\boldsymbol{\pi}_{n}(q)$ will serve as the base clause to an iterative $\mathcal{\varepsilon}$-scheme to compute better and better approximations to $\widehat{\Pi}$. This is the idea of SAPT (Panati et al., 2003).

SAPT uses an $\varepsilon$-scaled phase space (or deformation) quantization scheme (Blaszak and Domanski, 2012) for the slow subsector of the system, while retaining a standard Hilbert space representation for the fast sector. Phase space quantum mechanics is a formulation of quantum mechanics that employs an algebra of phase space functions $\mathcal{A}_{Q}$ instead of using the standard operator algebra in the Hilbert space representation of quantum mechanics. Quantum mechanical observables are thus represented by real-valued phase space functions. The pullback of the operator product to the phase space algebra gives rise to a non-commutative star product $\star$. Since the star product reduces to the commutative multiplication of phase space functions in the limit $\hbar \rightarrow 0$, this formulation of quantum mechanics is also known as deformation quantization. The standard textbooks by Dimassi and Sjöstrand (1999), Folland (1989), and Hörmander (1985a,b) give thorough introductions to the usual scalar-valued phase space quantization scheme and pseudodifferential calculus, but the situation here is more subtle. SAPT requires to consider operator-valued symbol functions, in particular functions on the slow phase space with values in the operators on the fast Hilbert space. We will thus deal with symbol functions of the form $\boldsymbol{A}(q, p) \in C^{\infty}\left(\Gamma_{s}, \mathcal{B}\left(\mathcal{H}_{\mathrm{f}}\right)\right)$ where $\Gamma_{\mathrm{s}}$ denotes the slow phase space (Teufel, 2003; Appendix A). It is straightforward to map the symbol functions (operator-valued or not) to their operator representatives in the standard Hilbert space approach. The concrete prescription depends of course on the operator ordering that one chooses. In case of the symmetric Weyl quantization prescription, this relation is provided by the Weyl correspondence (Dubin et al., 1980), and a symbol function appears as the kernel of an integral operator that acts on an element of the Hilbert space (Teufel, 2003).

Symbol functions which give rise to admissible operators in the quantum theory can be classified by their asymptotic behavior on the slow phase space. One important class of symbols relevant for SAPT are the semiclassical symbols $S_{\rho}^{m}$, with $m \in \mathbb{R}$ and $0 \geq \rho \geq 1$. An operator-valued function $A \in C^{\infty}\left(\mathbb{R}^{2}, \mathcal{B}\left(\mathcal{H}_{\mathrm{f}}\right)\right)$ is in the symbol class $S_{\rho}^{m}\left(\mathcal{B}\left(\mathcal{H}_{\mathrm{f}}\right)\right)$ if for every $\alpha, \beta \in \mathbb{N}$, there exists a positive constant $C_{\alpha, \beta}$ such that,

$$
\sup _{q \in \mathbb{R}}\left\|\left(\partial_{q}^{\alpha} \partial_{p}^{\beta} \boldsymbol{A}\right)(q, p)\right\|_{\mathcal{B}\left(\mathcal{H}_{\mathrm{f}}\right)} \leq C_{\alpha, \beta}\langle p\rangle^{m-\rho|\beta|},
$$

for every $p \in \mathbb{R}$, and $\langle p\rangle=\left(1+|p|^{2}\right)^{1 / 2}$ (Teufel, 2003). For such symbols the Weyl ordering prescription for quantum theory gives rise to a specific star product, and we can finally make sense of the space adiabatic perturbation idea. For two such operator-valued symbols on the slow phase space $\boldsymbol{A} \in S_{\rho}^{m_{1}}\left(\mathcal{B}\left(\mathcal{H}_{\mathrm{f}}\right)\right)$, $\boldsymbol{B} \in S_{\rho}^{m_{2}}\left(\mathcal{B}\left(\mathcal{H}_{\mathrm{f}}\right)\right)$, their star product is given by,

$(\boldsymbol{A} \star \boldsymbol{B})(q, P)=\left.\exp \left(\frac{i \hbar}{2}\left(\partial_{x} \partial_{P}-\partial_{q} \partial_{D}\right)\right) \boldsymbol{A}(x, D) \boldsymbol{B}(q, P)\right|_{x=q, D=P} \in S_{\rho}^{m_{1}+m_{2}}\left(\mathcal{B}\left(\mathcal{H}_{f}\right)\right)$.

We note that the exponential has a series expansion which could be considered as a series with respect to $\hbar$ in the given context. Alluding to the adiabaticity of the system, it is reasonable to define a rescaled momentum operator $p:=\varepsilon P$. Replacing $P$ and $D$ by their $\varepsilon$-scaled versions in the star product formula, the new expansion parameter is $\varepsilon \hbar$. Any star product of symbol functions can thus be written in a series expansion in $\varepsilon$. Comparing terms of the same polynomial order in $\varepsilon$, this defines a perturbation theory for quantum mechanical equations which will iteratively solve the eigenvalue problems of interest. The first two orders of the rescaled star product are given by,

$$
\begin{aligned}
(\boldsymbol{A} \star \boldsymbol{B})(q, p)= & \left(\boldsymbol{A}_{0} \cdot \boldsymbol{B}_{0}\right)(q, p)+\frac{i \varepsilon \hbar}{2}\left\{\boldsymbol{A}_{0}(q, p), \boldsymbol{B}_{0}(q, p)\right\} \\
& +\varepsilon\left(\boldsymbol{A}_{0} \cdot \boldsymbol{B}_{1}+\boldsymbol{A}_{1} \cdot \boldsymbol{B}_{0}\right)(q, p)+\mathcal{O}\left(\varepsilon^{2}\right),
\end{aligned}
$$

where the symbol functions have been expanded with respect to $\varepsilon$ according to $\boldsymbol{A}=\sum_{k} \varepsilon^{k} \boldsymbol{A}_{k}$ and $\boldsymbol{B}=\sum_{k} \varepsilon^{k} \boldsymbol{B}_{k}$. Note that the $\varepsilon$-rescaling changes the whole symplectic structure (we now have, $\left.\{q, p\}_{\mathrm{s}}=\varepsilon\right)$, as well as the canonical commutation relations since we obtain, 


$$
[\widehat{q}, \hat{p}]_{s}=i \varepsilon \hbar \widehat{1}_{s} .
$$

From now on, we will set $\hbar \equiv 1$. Note that in the original Born-Oppenheimer approximation, the perturbative parameter occurs (after an appropriate rescaling) only in the Hamiltonian and the perturbation theory consists in splitting the Hamiltonian (and its spectrum) accordingly, while here the quantum algebra is redefined, giving rise to a ( $n$ in principle infinite) perturbation series in $\varepsilon$.

\subsection{Space Adiabatic Perturbation Theory}

SAPT as introduced by Panati et al. (2003) places a set of conditions on the physical system under consideration. These are, in some respects, quite restrictive. However, if one accepts to abandon certain results, such as the convergence of the perturbative series, it is possible to milden the conditions. Here, we present the original conditions introduced by Panati, Spohn and Teufel for a system with $d$ slow and $k$ fast degrees of freedom, and which can be split into four categories:

(C1) The state space of the system decomposes as,

$$
\mathcal{H}=L^{2}\left(\mathbb{R}^{d}\right) \otimes \mathcal{H}_{\mathrm{f}}=L^{2}\left(\mathbb{R}^{d}, \mathcal{H}_{\mathrm{f}}\right),
$$

where $L^{2}\left(\mathbb{R}^{d}\right)$ is the state space of the system whose rate of change is by a factor $\varepsilon^{l}, l \in \mathbb{R}^{+}$, smaller than the rate of change of the (environmental) system $\mathcal{H}_{\mathrm{f}}$. The latter is assumed to be a separable Hilbert space.

(C2) The quantum Hamiltonian $\widehat{\boldsymbol{H}}$ (may it be an operator or a constraint) is given as the Weyl quantization of a semiclassical symbol $\quad \boldsymbol{H} \in S_{\rho}^{m}\left(\varepsilon, \mathcal{B}\left(\mathcal{H}_{\mathrm{f}}\right)\right), \quad$ i.e., $\quad \boldsymbol{H}$ asymptotically approaches an $\varepsilon$-series,

$$
\boldsymbol{H}(\varepsilon, z) \asymp \sum_{j=0}^{\infty} \varepsilon^{j} \boldsymbol{H}_{j}(z),
$$

where $\boldsymbol{H}_{j} \in S_{\rho}^{m-j \rho}\left(\mathcal{B}\left(\mathcal{H}_{\mathrm{f}}\right)\right)$ for all $j \in \mathbb{N}$ and $z:=(q, P) \in \mathbb{R}^{2 d}$. The appropriate notion of convergence is provided by a Fréchet semi-norm in $S_{\rho}^{m}\left(\mathcal{B}\left(\mathcal{H}_{\mathrm{f}}\right)\right.$ ), see (Teufel, 2003) for further details.

(C3) For any fixed $z \in \mathbb{R}^{2 d}$, the spectrum $\sigma(z)$ of the principal symbol $\boldsymbol{H}_{0}(z)$ of $\boldsymbol{H}(\varepsilon, z)$ has isolated parts $\sigma_{n}(z), n \in \mathbb{N}$. Picking one such $v \in \mathbb{N}$ and therefore suppressing any $n$-dependence in the following, the minimal distance between the elements of $\sigma_{v}(z)$ and the remainder of the spectrum $\sigma_{\text {rem }}(z):=\sigma(z) \backslash \sigma_{\nu}(z)$ displays a non-vanishing gap. According to its characteristics with varying $z$, the gap can be classified by means of a parameter $\gamma$.

Conditions $(\mathbf{G a p})_{\gamma}$ : Let $f_{ \pm} \in C^{0}\left(\mathbb{R}^{2 d}, \mathbb{R}\right)$ be two continuous functions with $f_{-} \leq f_{+}$.

(G1) Enclosing interval. For every $z \in \mathbb{R}^{2 d}$ the isolated part of the spectrum $\sigma_{n}(z)$ is entirely contained in the interval $I(z):=$ $\left[f_{-}(z), f_{+}(z)\right]$.
(G2) Gap to the remainder. The distance between the remainder of the spectrum, $\sigma_{\text {rem }}(z)$ and the enclosing interval $I(z)$ is strictly bigger than zero and increasing for large momenta, i.e.,

$$
\operatorname{Dist}\left[\sigma_{\text {rem }}(z), I(z)\right] \geq C_{\mathrm{g}}\left(1+p^{2}\right)^{\frac{\gamma}{2}} \text {. }
$$

(G3) Boundedness of the interval. The width of the interval $I(z)$ is uniformly bounded, i.e.,

$$
\sup _{z \in \mathbb{R}^{2 d}}\left|f_{+}(z)-f_{-}(z)\right| \leq C_{\mathrm{d}}<\infty \text {. }
$$

(C4) Convergence Condition. If the system satisfies the gap condition (C3) for some $\gamma \in \mathbb{R}$, the Hamilton symbol $\boldsymbol{H}$ must be in $S_{\rho}^{\gamma}$. If $\rho=0$, also $\gamma$ must vanish. If $\rho>0, \gamma$ can be any real number but $\widehat{\boldsymbol{H}}$ must be essentially self-adjoint on $\mathcal{S}\left(\mathbb{R}^{d}, \mathcal{H}_{\mathrm{f}}\right)$.

Condition (C4) is not vital in order to perform the formal computations in the following. It ensures however that for considerations on the whole slow phase space $\Gamma_{s}$, the error estimates of SAPT are bounded everywhere on $\Gamma_{s}$. In particular, the adiabatic decoupling is said to be uniform. Note also that the requirement that $\boldsymbol{H}$ has values in the bounded operators is violated for many physical systems of interest. In such cases, the space adiabatic scheme cannot be immediately applied and the convergence of the perturbative expansion has to be examined by independent methods (Panati et al., 2003).

Given the conditions $(\mathrm{C} 1)-(\mathrm{C} 4)$, the space adiabatic theorem introduces a perturbative construction scheme that is based on iteratively computing three symbol functions: the Moyal projector $\boldsymbol{\pi} \in S_{\rho}^{0}$, the Moyal unitary $\boldsymbol{u} \in S_{\rho}^{0}$ and an effective Hamiltonian $\boldsymbol{H}_{\text {eff }} \in S_{\rho}^{m}$. The Moyal projector serves to identify a subspace of the total Hilbert space which is almost invariant under the dynamics of $\widehat{\boldsymbol{H}}$ and which is associated with one particular quantum number $v \in \mathbb{N}$ of the fast sector. The Moyal unitary $\boldsymbol{u}$ is an auxiliary structure which gives rise to a unitary operator that maps the relevant subspace to a much simpler reference subspace. In fact, the original subspace is a technically complicated object and cannot provide us with a simple procedure to derive the (approximated) dynamics in the subspace. The reference subspace is trivial with respect to the fast subsystem and allows to compute the dynamics of the slow sector including the backreaction of the fast degree(s) of freedom. It is used to derive an effective Hamiltonian symbol $\boldsymbol{H}_{\text {eff }}$ whose solutions are approximate solutions to the full Hamilton operator $\widehat{\boldsymbol{H}}$. More precisely.

(S1) There exists a unique formal symbol, $\pi=\sum_{i \geq 0} \varepsilon^{i} \pi_{i}$, with $\boldsymbol{\pi}_{i} \in S_{\rho}^{-i \rho}\left(\mathcal{B}\left(\mathcal{H}_{\mathrm{f}}\right)\right)$, such that $\boldsymbol{\pi}_{0}$ is the spectral projection of $\boldsymbol{H}(q, p)$ corresponding to $\sigma_{\nu}(q, p)$ and with the properties,

$$
\begin{aligned}
& (\mathrm{S} 1-1): \boldsymbol{\pi} \star_{\varepsilon} \boldsymbol{\pi}=\boldsymbol{\pi} \quad(\mathrm{S} 1-2): \boldsymbol{\pi}^{\star}=\boldsymbol{\pi} \\
& (\mathrm{S} 1-3): \boldsymbol{H} \star_{\varepsilon} \boldsymbol{\pi}-\boldsymbol{\pi} \star_{\varepsilon} \boldsymbol{H}=0 .
\end{aligned}
$$


It can be shown that the Weyl quantization of a resummation of $\boldsymbol{\pi}$, which we denote by $\boldsymbol{\pi}_{\varepsilon}$ is $\mathcal{O}_{0}\left(\varepsilon^{\infty}\right)$-close to an operator $\hat{\boldsymbol{\Pi}}$, i.e., $\widehat{\boldsymbol{\Pi}}=\widehat{\boldsymbol{\pi}}_{\varepsilon}+\mathcal{O}_{0}\left(\varepsilon^{\infty}\right)$ and that, $[\widehat{\boldsymbol{H}}, \widehat{\boldsymbol{\Pi}}]=\mathcal{O}_{0}\left(\varepsilon^{\infty}\right)$.

(S2) Let $\pi_{\mathrm{R}}$ be the projection on some reference subspace $\mathcal{K}_{\mathrm{f}} \subseteq \mathcal{H}_{\mathrm{f}}$. We assume that there exists a symbol $\boldsymbol{u}_{0} \in S_{\rho}^{0}\left(\mathcal{B}\left(\mathcal{H}_{\mathrm{f}}\right)\right)$, such that, $\boldsymbol{u}_{0} \cdot \boldsymbol{\pi}_{0} \cdot \boldsymbol{u}_{0}^{*}=\boldsymbol{\pi}_{\mathrm{R}}$. Then, there is a formal symbol $\boldsymbol{u}=$ $\sum_{i \geq 0} \varepsilon^{i} \boldsymbol{u}_{i}$ with $\boldsymbol{u}_{i} \in S_{\rho}^{-i \rho}\left(\mathcal{B}\left(\mathcal{H}_{\mathrm{f}}\right)\right)$ such that,

$$
\begin{aligned}
& (\mathrm{S} 2-1): \boldsymbol{u}^{*} \star_{\varepsilon} \boldsymbol{u}=1 \quad(\mathrm{~S} 2-2): \boldsymbol{u} \star_{\varepsilon} \boldsymbol{u}^{*}=1 \\
& (\mathrm{~S} 2-3): \boldsymbol{u} \star_{\varepsilon} \boldsymbol{\pi} \star_{\varepsilon} \boldsymbol{u}^{*}=\boldsymbol{\pi}_{\mathrm{R}} .
\end{aligned}
$$

The Weyl quantization of a resummation of $\boldsymbol{u}$, which we denote by $\boldsymbol{u}_{\varepsilon}$ gives rise to an operator, $\widehat{\boldsymbol{U}}=\widehat{\boldsymbol{u}}_{\varepsilon}+\mathcal{O}_{0}\left(\varepsilon^{\infty}\right)$ for which it holds true that, $\hat{U} \widehat{\Pi} \hat{U}=\widehat{\pi}_{\mathrm{R}}$.

(S3) There exists a formal, effective Hamilton symbol $\boldsymbol{h}_{\text {eff }}=$ $\sum_{i \geq 0} \varepsilon^{i} \boldsymbol{h}_{\mathrm{eff}, i}$ defined as,

$$
\boldsymbol{h}_{\mathrm{eff}}:=\boldsymbol{u} \star_{\varepsilon} \boldsymbol{H} \star_{\varepsilon} \boldsymbol{u}^{*}
$$

For systems with an external time parameter $t$ and the Weyl quantizations $\widehat{\boldsymbol{u}}$ and $\widehat{\boldsymbol{h}}_{\text {eff }}$, we have,

$$
e^{-i \widehat{\boldsymbol{H}} s}-\widehat{\boldsymbol{u}}^{\dagger} e^{-i \widehat{\boldsymbol{h}}_{\mathrm{eff}} \boldsymbol{S}} \widehat{\boldsymbol{u}}=\mathcal{O}_{0}\left(\varepsilon^{\infty}|s|\right)
$$

In the next section, we will make these formal definitions and results more explicit and apply the space adiabatic scheme to a simple cosmological model up to second order in the perturbations.

\subsection{Backreaction in Quantum Cosmology}

As an illustrative example for the space adiabatic scheme, let us consider Einstein general relativity, reduced to spatial homogeneity and isotropy, including a cosmological constant $\Lambda>0$, and coupled to a spatially homogeneous, isotropic and real Klein-Gordon field $\phi_{0}$ with mass $m_{\mathrm{KG}}>0$ and coupling constant $\lambda \in \mathbb{R}$. We assume a globally hyperbolic space time manifold and a metric with Lorentzian signature $(-,+,+,+)$. The only dynamical degree of freedom of the metric is the scale factor $a \geq 0$. The lapse function $N$ is a Lagrange multiplier and will be fixed $N \equiv 1$. We perform a $(3+1)$-split of the manifold into space and time which admits spatial hypersurfaces $\sigma$ which we fix to be compact, flat three-tori $\mathbb{T}^{3}$ with side lengths $l \equiv 1$. The cosmological action is,

$$
S\left[a(t), \phi_{0}(t)\right]=\int_{\mathbb{R}} \mathrm{d} t\left(-\frac{1}{\kappa}\left(3 \dot{a}^{2} a+\Lambda a^{3}\right)+\frac{1}{2 \lambda}\left(\dot{\phi}_{0}^{2}-m_{\mathrm{KG}}^{2} \phi_{0}^{2}\right)\right),
$$

where $\kappa=8 \pi G$ and $\lambda$ are the gravitational and scalar field coupling constants. If both, $\left(a, \phi_{0}\right)$ are dimensionless, as we assume, then both coupling constants have the same dimension, and we define the dimensionless ratio,

$$
\varepsilon^{2}:=\frac{\kappa}{\lambda}
$$

Considering typical values of the coupling parameters in the Standard Model, it seems reasonable to assume that this ratio is indeed extremely small. Hence, we identify gravity with the slow sector while the matter field is considered to be the fast subsystem.

The space adiabatic scheme requires a Hamiltonian formulation of the problem. We define the conjugate momenta of $a$ and $\phi_{0}$ as, $p_{a}:=\varepsilon \frac{\partial L}{\partial a}$ and $\mu_{0}:=\frac{\partial L}{\partial \dot{\phi}_{0}}$, where $L$ is the Lagrange function associated with the action $S$. The Poisson brackets of the canonical variables evaluate to $\left\{a, p_{a}\right\}=\varepsilon$, and $\left\{\phi_{0}, \mu_{0}\right\}=1$. The Legendre transformation generates the Hamilton constraint,

$$
C\left(a, p_{a}, \phi_{0}, \pi_{0}\right):=-\frac{1}{12} \frac{p_{a}^{2}}{a}+\frac{\Lambda}{\lambda \kappa} a^{3}+\frac{\mu_{0}^{2}}{2 a^{3}}+\frac{1}{2 \lambda^{2}} m_{\mathrm{KG}}^{2} a^{3} \phi_{0}^{2}
$$

where for notational reasons, we divided the whole constraint by a constant factor $\lambda$. For simplifying the analysis by means of SAPT in the following, we switch to triad-like canonical variables,

$$
b:= \pm \sqrt{a^{3}}, \quad \rho:=\frac{2}{3} \frac{p_{a}}{\sqrt{a}}
$$

which is a double cover of the original phase space and we do not restrict to any of the branches of $b$. In order to keep the notation as simple as possible, we introduce the following parameters and functions,

$$
m_{\mathrm{G}}:=\frac{8}{3}, \quad \omega_{\mathrm{G}}^{2}:=\frac{3 \Lambda}{4 \lambda \kappa}, \quad \tilde{m}_{\mathrm{KG}}:=b^{2}, \quad \omega_{\mathrm{KG}}^{2}:=\frac{m_{\mathrm{KG}}^{2}}{\lambda^{2}} .
$$

These definitions and the new canonical variables give for the Hamilton constraint,

$$
\begin{aligned}
C\left(b, \rho, \phi_{0}, \mu_{0}\right)=- & \frac{\rho^{2}}{2 m_{\mathrm{G}}}+\frac{1}{2} m_{\mathrm{G}} \omega_{\mathrm{G}}^{2} b^{2}+\frac{\mu_{0}^{2}}{2 \tilde{m}_{\mathrm{KG}}(b)} \\
& +\frac{1}{2} \tilde{m}_{\mathrm{KG}}(b) \omega_{\mathrm{KG}}^{2} \phi_{0}^{2} .
\end{aligned}
$$

We quantize the system and start by considering the scalar field subsystem using bold operator symbols. The state space is $\mathcal{H}_{\mathrm{f}}:=L^{2}\left(\mathbb{R}, \mathrm{d} \phi_{0}\right)$, and the scalar field operator and its conjugate momentum satisfy the canonical commutation relation, $\left[\phi_{0}, \boldsymbol{\mu}_{0}\right]_{\mathrm{f}}=i 1_{\mathrm{f}}$. Similarly, the state space of the geometrical subsystem is $\mathcal{H}_{s}:=L^{2}(\mathbb{R}, \mathrm{d} b)$. The quantum operators wear hats and the canonical commutation relation for the geometrical variable and its conjugate momentum are, $[\widehat{b}, \hat{\rho}]_{s}=i \varepsilon \hat{1}_{s}$. The quantum theory of the coupled system has the tensor product Hilbert space, $\mathcal{H}=\mathcal{H}_{\mathrm{s}} \otimes \mathcal{H}_{\mathrm{f}}$. The constraint operator on $\mathcal{H}$ is given by,

$$
\begin{aligned}
\widehat{\boldsymbol{C}}=( & \left.-\frac{\hat{\rho}^{2}}{2 m_{\mathrm{G}}}+\frac{1}{2} m_{\mathrm{G}} \omega_{\mathrm{G}}^{2} \widehat{b}^{2}\right) \otimes \mathbf{1}_{\mathrm{f}}+\frac{1}{2 \tilde{m}_{\mathrm{KG}}(\widehat{b})} \otimes \boldsymbol{\mu}_{0}^{2} \\
& +\frac{1}{2} \tilde{m}_{\mathrm{KG}}(\widehat{b}) \omega_{\mathrm{KG}}^{2} \otimes \phi_{0}^{2} .
\end{aligned}
$$

We check the conditions (C1)-(C4) for SAPT. (C1) holds without further ado since the cosmological Hilbert space $\mathcal{H}_{\mathrm{s}} \otimes \mathcal{H}_{\mathrm{f}}$ has the required tensor product structure, and $\mathcal{H}_{\mathrm{s}}$ is an $L^{2}$-space and $\mathcal{H}_{\mathrm{f}}$ is separable. We represent the quantum 
constraint as a symbol function $\boldsymbol{C}(\rho, b)$ with values in the linear operators on the Klein-Gordon Hilbert space $\mathcal{H}_{\mathrm{f}}$ by formally quantizing the Klein-Gordon subsystem only,

$$
\begin{aligned}
\boldsymbol{C}(b, \rho)= & \left(-\frac{\rho^{2}}{2 m_{\mathrm{G}}}+\frac{1}{2} m_{\mathrm{G}} \omega_{\mathrm{G}}^{2} b^{2}\right) 1_{\mathrm{f}}+\frac{\phi_{0}^{2}}{2 \tilde{m}_{\mathrm{KG}}(b)} \\
& +\frac{1}{2} \tilde{m}_{\mathrm{KG}}(b) \omega_{\mathrm{KG}}^{2} \boldsymbol{\mu}_{0}^{2} .
\end{aligned}
$$

$C(b, \rho)$ is an unbounded operator on $\mathcal{H}_{\mathrm{f}}$ for every $(b, \rho) \in \mathbb{R}^{2}$. In particular, the operator corresponds to the Hamiltonian of a quantum harmonic oscillator with constant frequency $\omega_{\mathrm{KG}}$, $b$-dependent mass $\tilde{m}_{\mathrm{KG}}(b)$ and an off-set energy. As such, the symbol has for fixed finite $(b, \rho)$ an energy spectrum which is bounded from below but not from above. Besides, $C(b, \rho)$ is an unbounded function with respect to both, $b$ and $\rho$. According to SAPT, the constraint symbol must however belong to one of the symbol classes $S_{\rho}^{m}\left(\mathcal{B}\left(\mathcal{H}_{\mathrm{f}}\right)\right)$ and should therefore have values in the space of bounded operators on $\mathcal{H}_{\mathrm{f}}$, be a bounded function with respect to $b$ and grow maximally polynomially in $\rho$. By means of the standard quantum oscillator eigensolutions $\xi_{n} \in \mathcal{H}_{\mathrm{f}}$, $n \in \mathbb{N}$ with a $b$-dependent mass, the correspondent eigenvalue equation has the form,

$$
\begin{aligned}
& C(b, \rho) \xi_{n}(b)=E_{n}(b, \rho) \xi_{n}(b), \\
& E_{n}(b, \rho)=-\frac{\rho^{2}}{2 m_{\mathrm{G}}}+\frac{1}{2} m_{\mathrm{G}} \omega_{\mathrm{G}}^{2} b^{2}+\omega_{\mathrm{KG}}\left(n+\frac{1}{2}\right)
\end{aligned}
$$

We emphasize that the $b$-dependence of the states is purely parametric which allows to define $b$-dependent projection operators on $\mathcal{H}_{\mathrm{f}}$,

$$
\pi_{n}(b):=\xi_{n}(b)\left\langle\xi_{n}(b), \cdot\right\rangle_{\mathcal{H}_{\mathrm{f}}},
$$

by means of which the Hamilton symbol constraint has the spectral representation,

$$
C(b, \rho)=\sum_{n \geq 0} E_{n}(b, \rho) \boldsymbol{\pi}_{n}(b) .
$$

In order to respect the conditions for the application of SAPT, it is possible to define an auxiliary Hamilton symbol $\boldsymbol{C}_{\text {aux }}(b, \rho)$ which has values in the bounded operators, is locally a bounded function with respect to $b$, and which preserves the local structure of the symbol function $\boldsymbol{C}(b, \rho)$ (Panati et al., 2003; Stottmeister, 2015). Since the perturbation scheme is applicable without referring to this auxiliary symbol (if convergence and uniformity of the series expansion do not play a role for the time being), we continue working with the original symbol (32) to illustrate the scheme. Most importantly, the gap condition (C3) is satisfied since the energy functions $E_{n}(b, \rho)$ are gapped functions on the gravitational phase space. Finally, we formally choose one of the fast energy bands with quantum number $v \in \mathbb{N}$ to proceed with the space adiabatic scheme.

\section{Application of Space Adiabatic Perturbation Theory}

We start with the perturbative construction of the Moyal projector symbol $\pi$ up to first order in $\varepsilon$. In fact, this will be sufficient to define the effective Hamilton constraint up to second order. With the ansatz, $\boldsymbol{\pi}_{(1)}=\pi_{0}+\varepsilon \pi_{1}$, and the natural choice for the base clause,

$$
\pi_{0}:=\xi_{v}(b)\left\langle\xi_{v}(b), \cdot\right\rangle_{\mathcal{H}_{\mathrm{f}}},
$$

we construct the symbol function $\pi_{(1)}(b, \rho)$ following the construction steps (S1). The first condition (S1-1), $\pi \star_{\varepsilon} \boldsymbol{\pi}=\boldsymbol{\pi}$, yields that the diagonal contribution to $\pi_{1}$ vanishes because $\pi_{0}(b)$ depends solely on $b$. Regarding the third condition (S1-3), $\boldsymbol{C}_{0} \star_{\varepsilon} \boldsymbol{\pi}-\boldsymbol{\pi} \star_{\varepsilon} \boldsymbol{C}_{0}=0$, it is straightforward to derive (Teufel, 2003; Neuser et al., 2019), that,

$$
\begin{aligned}
\boldsymbol{\pi}_{1}= & -\frac{i}{2} \boldsymbol{\pi}_{0} \cdot\left\{\boldsymbol{\pi}_{0}, \boldsymbol{C}_{0}+E_{\nu} 1_{\mathrm{f}}\right\}_{\mathrm{s}} \cdot\left(\boldsymbol{C}_{0}^{\perp}-E_{\nu} 1_{\mathrm{f}}\right)^{-1} \cdot \boldsymbol{\pi}_{0}^{\perp} \\
& -\frac{i}{2}\left(\boldsymbol{C}_{0}^{\perp}-E_{\nu} 1_{\mathrm{f}}\right)^{-1} \cdot \boldsymbol{\pi}_{0}^{\perp} \cdot\left\{\boldsymbol{C}_{0}+E_{\nu} 1_{\mathrm{f}}, \boldsymbol{\pi}_{0}\right\}_{\mathrm{s}} \cdot \boldsymbol{\pi}_{0}
\end{aligned}
$$

as a determining equation for $\boldsymbol{\pi}_{1}$, where we defined, $\boldsymbol{C}_{0}^{\perp}=\boldsymbol{C}_{0} \cdot \boldsymbol{\pi}_{0}^{\perp}$, and $\pi_{0}^{\perp}:=1_{\mathrm{f}}-\pi_{0}$. To evaluate the partial derivative $\partial_{b} \pi_{0}$ in this equation, we need to evaluate the derivative of the states $\xi_{n}(b) \in \mathcal{H}_{\mathrm{f}}$ as well as the derivatives of the canonically defined creation and annihilation operators $\boldsymbol{a}^{*}(b) \in \mathcal{L}\left(\mathcal{H}_{\mathrm{f}}\right)$ and $\boldsymbol{a}(b) \in \mathcal{L}\left(\mathcal{H}_{\mathrm{f}}\right)$. Therefore, recall that the initial eigenvalue problem admits the oscillator solutions $\xi_{n}(b)$. Accordingly, the creation operator $\boldsymbol{a}^{*}(b)$ can be written in terms of the canonical pair $\left(\phi_{0}, \boldsymbol{\mu}_{0}\right)$ as,

$$
\boldsymbol{a}^{*}(b)=\sqrt{\frac{\tilde{m}_{\mathrm{KG}}(b) \omega_{\mathrm{KG}}}{2}}\left(\phi_{0}-\frac{i}{\tilde{m}_{\mathrm{KG}}(b) \omega_{\mathrm{KG}}} \boldsymbol{\mu}_{0}\right),
$$

The derivatives of the vacuum state $\xi_{0}(b)$ and the creation operator are given by,

$$
\frac{\partial \xi_{0}}{\partial b}:=\sqrt{2} f(b) \xi_{2}(b), \quad \frac{\partial \boldsymbol{a}^{*}(b)}{\partial b}=-2 f(b) \boldsymbol{a}(b),
$$

where $f(b):=-\left(\partial_{b} \tilde{m}_{\mathrm{KG}}\right) /\left(4 \tilde{m}_{\mathrm{KG}}\right)=-1 /(2 b)$. We propose the definition of a covariant derivative, or more precisely, a gauge potential $\mathcal{A}$, associated with the $b$-derivative of the fast oscillator states. Note that this is simply Berry's connection (Berry, 1984). Using the natural basis choice from above, its coefficients with respect to the $b$-direction on $\Gamma_{\mathrm{s}}$ are given by,

$$
\frac{\partial \xi_{n}(b)}{\partial b}=\mathcal{A}_{b n}^{n-2}(b) \xi_{n-2}(b)+\mathcal{A}_{b n}^{n+2}(b) \xi_{n+2}(b)
$$

with, $\quad \mathcal{A}_{b n}^{k}(b)=-\sqrt{n(n-1)} f(b) \delta_{n}^{k+2}+\sqrt{(n+1)(n+2)} f(b)$ $\delta_{n}^{k-2}$. All coefficients $\mathcal{A}_{\rho n}{ }^{m}$ in the $\rho$-direction vanish because the fast eigenstates do not depend on $\rho$. Only the coefficients that connect states differing by two excitations in the $b$-direction are nonvanishing. Since we have real-valued eigenstates, the connection coefficients are real-valued, too, such that the orthonormality relation between the fast states yields that, $\mathcal{A}_{b n}{ }^{m}=-\mathcal{A}_{b m}{ }^{n}$. The $b$-derivative of the projector symbol $\boldsymbol{\pi}_{0}$ follows from using Riesz' representation theorem and one can simply write,

$$
\frac{\partial \pi_{0}}{\partial b}=\mathcal{A}_{b v}^{m}\left(\xi_{\nu}\left\langle\xi_{m}, \cdot\right\rangle_{\mathrm{f}}+\xi_{m}\left\langle\xi_{v}, \cdot\right\rangle_{\mathrm{f}}\right),
$$

where $v$ is still a fixed quantum number while $m$ runs over all natural numbers. To evaluate $\pi_{1}$, the partial derivative, 
$\partial_{\rho}\left(C_{0}+E_{v} \cdot 1_{\mathrm{f}}\right)$, is simply $\left(-2 \rho / m_{\mathrm{G}}\right) \cdot 1_{\mathrm{f}}$, because only the spectral functions $E_{n}(b, \rho)$ depend on $\rho$ while the states do not. The functional form of the energy functions also reduces $\left(C_{0}^{\perp}-E_{\nu} \cdot 1_{\mathrm{f}}\right)$ to a factor $\pm\left(2 \omega_{\mathrm{KG}}\right)^{-1}$, and consequently,

$$
\begin{aligned}
\pi_{1}=-\frac{i \rho}{2 m_{\mathrm{G}} \omega_{\mathrm{KG}}} & \left(\mathcal{A}_{b v}{ }^{\nu-2}\left(\xi_{v}\left\langle\xi_{v-2}, \cdot\right\rangle_{\mathrm{f}}-\xi_{v-2}\left\langle\xi_{v}, \cdot\right\rangle_{\mathrm{f}}\right)\right. \\
& \left.+\mathcal{A}_{b v}^{v+2}\left(\xi_{\nu+2}\left\langle\xi_{v}, \cdot\right\rangle_{\mathrm{f}}-\xi_{\nu}\left\langle\xi_{v+2}, \cdot\right\rangle_{\mathrm{f}}\right)\right) .
\end{aligned}
$$

One can easily check that $\boldsymbol{\pi}_{(1)}$ satisfies all three conditions subsumed under (S1) up to first order in $\varepsilon$, i.e., that it is a projector and commutes with the full Hamilton symbol up to errors of order $\varepsilon^{2}$. We see that the improved projection symbol mixes adjacent eigenstates of the fast system, and going to higher orders in the perturbative scheme more and more states will be included.

The next step of SAPT consists in constructing the unitary symbol $\boldsymbol{u}_{1}$ which maps the dynamical subspace related to $\boldsymbol{\pi}_{(1)}$, to a suitable reference subspace $\mathcal{K}_{\mathrm{f}} \subset \mathcal{H}_{\mathrm{f}}$. It is convenient to choose one point $\left(b_{0}, \rho_{0}\right) \in \Gamma_{\mathrm{s}}$ and define the reference projection as,

$$
\pi_{\mathrm{R}}:=\xi_{\nu}\left(b_{0}\right)\left\langle\xi_{\nu}\left(b_{0}\right), \cdot\right\rangle_{\mathrm{f}}=: \zeta_{\nu}\left\langle\zeta_{\nu}, \cdot\right\rangle_{\mathrm{f}},
$$

where $\zeta_{v} \in \mathcal{H}_{\mathrm{f}}$ does not depend on the gravitational phase space variables. In a similar fashion, one can define the complete basis $\zeta_{n}:=\xi_{n}\left(b_{0}\right), n \in \mathbb{N}$ at the point $b_{0}$. A natural choice for the unitary operator in line with conditions (S2) at zeroth order is simply,

$$
\boldsymbol{u}_{0}(b)=\sum_{n \geq 0} \zeta_{n}\left\langle\xi_{n}(b), \cdot\right\rangle_{\mathrm{f}}
$$

In order to construct $\boldsymbol{u}_{1}$, the scheme splits the symbol into a hermitian and an antihermitian part. The hermitian part is determined by Eqs. S2-1 and S2-2, namely by requiring that $u \star u^{\star}=1_{\mathrm{f}}$ holds up to first order in $\varepsilon$. Since $\boldsymbol{u}_{0}$ only depends on the configuration variable $b$, the hermitian part vanishes trivially. The antihermitian part is determined by restricting equation (S2S-equation (S3), i.e., $\boldsymbol{u} \star \boldsymbol{\pi} \star \boldsymbol{u}^{\star}=\boldsymbol{\pi}_{\mathrm{R}}$, to the first order. It yields for $\boldsymbol{u}_{1}$ (Neuser et al., 2019),

$$
\begin{aligned}
\boldsymbol{u}_{1}= & {\left[\boldsymbol{\pi}_{\mathrm{R}}, \boldsymbol{u}_{0} \cdot \boldsymbol{\pi}_{1}^{\mathrm{OD}} \cdot \boldsymbol{u}_{0}^{*}\right]_{\mathrm{f}} \cdot \boldsymbol{u}_{0} } \\
=\frac{i \rho}{2 m_{\mathrm{G}} \omega_{\mathrm{KG}}} & {\left[\mathcal{A}_{b \nu}^{v-2}\left(\zeta_{\nu}\left\langle\xi_{v-2}, \cdot\right\rangle_{\mathrm{f}}+\zeta_{\nu-2}\left\langle\xi_{v}, \cdot\right\rangle_{\mathrm{f}}\right)\right.} \\
& \left.\quad-\mathcal{A}_{b v}^{v+2}\left(\zeta_{\nu+2}\left\langle\xi_{\nu}, \cdot\right\rangle_{\mathrm{f}}+\zeta_{\nu}\left\langle\xi_{\nu+2}, \cdot\right\rangle_{\mathrm{f}}\right)\right] .
\end{aligned}
$$

Eventually, we are ready to compute the effective Hamiltonian symbol up to second order in the perturbations, and which we restrict to the selected reference space, i.e., we compute, $\boldsymbol{C}_{\text {eff, (2),R }}(b, \rho):=\boldsymbol{\pi}_{\mathrm{R}} \cdot \boldsymbol{C}_{\text {eff,(2) }}(b, \rho) \cdot \boldsymbol{\pi}_{\mathrm{R}}$. The zeroth order contribution of this symbol is given according to condition (S3) by,

$$
\boldsymbol{C}_{\mathrm{eff}, 0, \mathrm{R}}(b, \rho)=\left(-\frac{\rho^{2}}{2 m_{\mathrm{G}}}+\frac{1}{2} m_{\mathrm{G}} \omega_{\mathrm{G}}^{2} b^{2}+\omega_{\mathrm{KG}}\left(\nu+\frac{1}{2}\right)\right) \boldsymbol{\pi}_{\mathrm{R}} .
$$

Thus, the effective constraint symbol for the gravitational degrees of freedom includes the bare gravitational constraint symbol plus an off-set energy which stems from the energy band associated with the quantum number $v$ of the Klein-Gordon system. This result corresponds to the Born-Oppenheimer approximation.
The first order contribution of the effective constraint symbol, $C_{\text {eff, } 1}(b, \rho)$ contains only off-diagonal terms, such that $C_{\text {eff, } 1, \mathrm{R}}(b, \rho)$ vanishes identically,

$$
\boldsymbol{C}_{\text {eff }, 1, \mathrm{R}}(b, \rho)=\frac{i}{2}\left\{\boldsymbol{\pi}_{\mathrm{R}} \cdot \boldsymbol{u}_{0}, \boldsymbol{C}_{0}+E_{\nu} 1_{\mathrm{f}}\right\}_{\mathrm{s}} \cdot \boldsymbol{u}_{0}^{*} \cdot \boldsymbol{\pi}_{\mathrm{R}}=0 .
$$

The same reasoning applies to the computation of the second order contribution $C_{\mathrm{eff}, 2, \mathrm{R}}(b, \rho)$ giving,

$$
\begin{aligned}
& \boldsymbol{C}_{\mathrm{eff}, 2, \mathrm{R}}=\frac{i}{2}\left\{\boldsymbol{\pi}_{\mathrm{R}} \cdot \boldsymbol{u}_{1}, \boldsymbol{C}_{0}+E_{\nu} 1_{\mathrm{f}}\right\}_{\mathrm{s}} \cdot \boldsymbol{u}_{0} \cdot \boldsymbol{\pi}_{\mathrm{R}} \\
& =\left[\frac{\partial E_{n}}{\partial \rho}\right]^{2}\left[\frac{\left(\mathcal{A}_{b \nu}^{v-2}\right)^{2}}{E_{\nu}-E_{\nu-2}}+\frac{\left(\mathcal{A}_{b v}^{v+2}\right)^{2}}{E_{v}-E_{v+2}}\right] \boldsymbol{\pi}_{\mathrm{R}}+\frac{1}{2} \frac{\partial^{2} E_{n}}{\partial \rho^{2}}\left[\left(\mathcal{A}_{b \nu}^{v-2}\right)^{2}+\left(\mathcal{A}_{b \nu}^{v+2}\right)^{2}\right] \boldsymbol{\pi}_{\mathrm{R}} .
\end{aligned}
$$

Finally, inserting the explicit results for the energy functions and the connection coefficients yields,

$$
\boldsymbol{C}_{\mathrm{eff}, 2, \mathrm{R}}(b, \rho)=-\frac{1}{2 m_{\mathrm{G}}}\left(\frac{\rho^{2}}{m_{\mathrm{G}} \omega_{\mathrm{KG}} b^{2}}\left(\nu+\frac{1}{2}\right)+\frac{1}{2 b^{2}}\left(v^{2}+v+1\right)\right) \boldsymbol{\pi}_{\mathrm{R}} .
$$

This proves our statement that besides the trivial Born-Oppenheimer approximation, further backreaction effects arise for the gravitational subsystem. It is now easy to evaluate the action of this symbol on some generic tensor product wave function in $\mathcal{H}=\mathcal{H}_{\mathrm{s}} \otimes \mathcal{H}_{\mathrm{f}}$, since the Klein-Gordon tensor factor does not depend on the gravitational degrees of freedom anymore. The problem reduces to a quantum constraint equation on $\mathcal{H}_{\mathrm{s}}$ only, and can be studied for each $v$ of interest. Nevertheless, we point out that finding states that are annihilated by the constraint operator $\widehat{\boldsymbol{C}}_{\text {eff }, 2, \mathrm{R}}$ is not a trivial task as it depends non-polynomially on b. Further details can be found in (Neuser et al., 2019). Finally, the question is how the solutions of (46) relate to the solutions of the original problem on $\mathcal{H}$. In fact, one needs to rotate the solutions of $\widehat{\boldsymbol{C}}_{\text {eff,2,R }}$ back to the original Hilbert space using the quantization of the Moyal unitary. It turns out that the resulting solutions are also approximate (orthogonal) solutions to the full Hamilton constraint at the respective perturbative order (Teufel, 2003; Schander and Thiemann, 2019a).

\subsection{Backreaction in Inhomogeneous Quantum Cosmology}

The purely homogeneous model in the previous section can serve as a showcase for a more realistic inhomogeneous cosmological model. Here, we consider standard cosmological perturbation theory for a gravitational metric field $g$, a massive real scalar field $\Phi$ as the matter content, and a cosmological constant, $\Lambda>0$. After the split of the relevant degrees of freedom into a homogeneous and an inhomogeneous part, the aim will be to incorporate backreactions from the perturbative degrees of freedom onto the homogeneous and isotropic background degrees of freedom.

As before, the model rests on a four-dimensional globally hyperbolic space time manifold $\mathcal{M}$ that admits the time space split $\mathcal{M} \cong \mathbb{R} \times \sigma$. The metric has Lorentzian signature $(-,+,+,+)$, and the spatial hypersurfaces $\sigma$ are compact and 
flat three-tori $\sigma \cong \mathbb{T}^{3}$ with side lenghts $l \equiv 1$. The global time parameter $t \in \mathbb{R}$ labels the spatial Cauchy surfaces $\Sigma_{t} . n^{\mu}$ is the unit normal vector field to these hypersurfaces, $N$ and $N_{\mu}$ the (standard) lapse and shift functions which parametrize the normal and the tangential part of the foliation. The task of specifying constraints or equations of motion for the metric field $g$, translates into defining a Cauchy initial value problem for the spatial metric $h_{\mu \nu}=g_{\mu \nu}+n_{\mu} n_{\nu}$ induced by $g$. The extrinsic curvature associated with the time derivative of $h$ is given by $K_{\mu \nu}=h_{\mu}^{\rho} h_{\nu}^{\lambda} \nabla_{\rho} n_{\lambda}$. $\nabla$ is the unique, torsion-free covariant derivative associated with the metric $g$. After pulling back the tensor fields to $\mathbb{R} \times \mathbb{T}^{3}$ and denoting spatial indices on the spatial hypersurfaces with lower case latin symbols, $a, b, c, \ldots \in\{1,2,3\}$, the Lagrange density is expressed by the sum of the Einstein-Hilbert Lagrange density $\mathcal{L}_{\mathrm{EH}}$ and the scalar field Lagrange density $\mathcal{L}_{\Phi}$, with,

$$
\begin{gathered}
\mathcal{L}_{\mathrm{EH}}=\frac{1}{2 \kappa} \sqrt{|h|} N\left(R^{(3)}+K_{a b} K^{a b}-\left(K_{a}^{a}\right)^{2}-2 \Lambda\right) \\
\mathcal{L}_{\Phi}=\frac{1}{2 \lambda} \sqrt{|h|} N\left(-\frac{1}{N^{2}} \dot{\Phi}^{2}+2 \frac{N^{a}}{N^{2}} \dot{\Phi} \partial_{a} \Phi+\left(h^{a b}-\frac{N^{a} N^{b}}{N^{2}}\right) \partial_{a} \Phi \partial_{b} \Phi\right. \\
\left.+m_{\Phi}^{2} \Phi^{2}\right) .
\end{gathered}
$$

where again, $\lambda$ is the coupling constant of the scalar field, $m_{\Phi}$ is the mass parameter of the scalar field, and $R^{(3)}$ is the curvature scalar associated with the three-metric $h$ and its Levi-Civita covariant derivative, $D$. The only degrees of freedom of the spatially homogeneous and isotropic sector are the zeroth order lapse function $N_{0}(t)$ and the scale factor $a(t)$, associated with the zeroth order spatial metric, ${ }^{0} h(t, x):=a^{2}(t)^{0} \tilde{h}(x)$ with ${ }^{0} \tilde{h}(x)$ being the time-independent metric on the spatial hypersurfaces. A Hamiltonian analysis shows that the lapse function is a Lagrange multiplier with no dynamical features, affirming the arbitrariness of the hypersurface foliation.

We introduce perturbations of the homogeneous degrees of freedom using a decomposition into scalar, vector and tensor parts (Halliwell and Hawking, 1985). Since we make use of their results in a later stage, we will stick to the definition of perturbations used by Castelló Gomar et al. (2015) and Martínez and Olmedo (2016),

$$
\begin{gathered}
N(t, x)=: N_{0}(t)+a^{3}(t) g(t, x) \\
N_{a}(t, x)=: a^{2}(t) D_{a} k(t, x)+a^{2}(t) \epsilon_{a}^{b c} D_{b} k_{c}(t, x) \\
h_{a b}(t, x)=: a^{2}(t)\left[(1+2 \alpha(t, x))^{0} \tilde{h}_{a b}(x)+6\left(D_{a} D_{b}-\frac{1}{3}{ }^{0} \tilde{h}_{a b}(x) D_{c} D^{c}\right) \beta(t, x)\right. \\
\left.+2 \sqrt{6} t_{a b}(t, x)+4 \sqrt{3} D_{(a} v_{b)}(t, x)\right] \\
\Phi(t, x)=: \phi(t)+\varphi(t, x) .
\end{gathered}
$$

where we introduced the perturbative scalar fields $(g, k, \alpha, \beta, \varphi)$, the vector fields $v_{a}$ and $k_{a}$, and the tensor field perturbations $t_{a b}$. For notational reasons, we introduce the fields $\breve{k}:=\Delta k$ and $\check{k}_{a}:=\epsilon_{a}^{b c} D_{b} k_{c}$ as new degrees of freedom associated with the shift.
We perform a Legendre transformation to obtain the Hamilton constraint. We insert the perturbed variables from Eqs. 49-(52) into the Lagrange density (47), (48), and expand the Lagrangian and the action functional $S$ up to second order in the perturbations. As the three-torus does not have a boundary, total divergences vanish in the computations. The resulting action does neither depend on the velocities of the lapse variables $N_{0}$ and $g$, nor on the velocities of the shift variables $\breve{k}$ and $\check{k}_{a}$. These Lagrange multipliers will hence be associated with primary constraint equations in the Hamiltonian formalism. In the lines of (Castelló Gomar et al., 2015), we define the conjugate momenta $\left(P_{a}, P_{\phi}\right)$ for the homogeneous and isotropic degrees of freedom $(a, \phi)$ using the Lagrange function $L=\int \mathrm{d} x \mathcal{L}$,

$$
P_{a}:=\frac{\partial L}{\partial \dot{a}}=-\frac{6}{\kappa N} a \dot{a}, \quad P_{\phi}:=\frac{\partial L}{\partial \dot{\phi}}=\frac{a^{3}}{\lambda N} \dot{\phi} .
$$

We denote the corresponding phase space by $\Gamma_{\text {hom }}=\Gamma_{s}$. The perturbation fields $\left(\alpha, \beta, \varphi, \vartheta_{a}, t_{a b}\right)$ together with their conjugate momenta $\left(\pi_{\alpha}, \pi_{\beta}, \pi_{\varphi}, \pi_{v}^{a}, \pi_{t}^{a b}\right)$ span the perturbative phase space $\Gamma_{\text {pert }}=\Gamma_{\mathrm{f}}$. The momenta are defined as,

$$
\pi_{\chi}:=\frac{\partial \mathcal{L}}{\partial \dot{\chi}}
$$

for any field $\chi \in\left\{\alpha, \beta, \varphi, v_{a}, t_{a b}\right\} . N_{0}, g, \check{k}$ and $\check{k}_{a}$ induce the lapse and shift primary constraints $\Pi_{0}^{N_{0}}, \Pi_{1}^{g}, \Pi_{1}^{k}$ and $\Pi_{1}^{k_{a}, b}$. The Hamiltonian density is eventually given by,

$$
\begin{gathered}
\mathcal{H}=N_{0}\left[\mathcal{H}_{0}+\mathcal{H}_{2}^{\mathrm{s}}+\mathcal{H}_{2}^{v}+\mathcal{H}_{2}^{t}\right]+g \cdot \mathcal{H}_{1}^{g}+\check{k}_{a} \cdot \mathcal{H}_{1}^{\check{k}_{d}, a}+\check{k} \cdot \mathcal{H}_{1}^{\check{k}^{+}} \\
\lambda_{N_{0}} \cdot \Pi_{0}^{N_{0}}+\lambda_{g} \cdot \Pi_{1}^{g}+\lambda_{\breve{k}} \cdot \Pi_{1}^{\check{k}}+\lambda_{\check{k}_{a}, b} \cdot \Pi_{1}^{\check{k}_{a}, b}
\end{gathered}
$$

$\mathcal{H}_{0}$ denotes the Hamiltonian contribution associated with the completely homogeneous and isotropic model. $\mathcal{H}_{2}^{\mathrm{s}}, \mathcal{H}_{2}^{v}$ and $\mathcal{H}_{2}^{t}$ are of second order in the perturbations and contain only scalar, vector and tensor variables respectively. $\mathcal{H}_{1}^{g}, \mathcal{H}_{1}^{\mathfrak{k}_{d}, a}$ and $\mathcal{H}_{1}^{\grave{k}}$ represent first order contributions which factorize with the respective lapse and shift variables. The second line lists the primary constraints associated with lapse and shift and their Lagrange multipliers $\lambda_{N_{0}}, \lambda_{g}, \lambda_{\check{k}}$ and $\lambda_{\check{k}_{a}, b}$. The system is completely constrained and we thus perform a Dirac analysis to extract the relevant physics.

Therefore, we identify a suitable set of free variables-in fact, the Dirac analysis will then become a trivial task. We start by noting that the perturbation variables that we introduced are not all gauge-invariant. In the scalar sector, let us employ the gauge-invariant Mukhanov-Sasaki variable $\vartheta$ (Mukhanov, 1988, 2005),

$$
\vartheta:=a \varphi+\frac{6 \lambda P_{\phi}}{\kappa P_{a}}(\alpha-\Delta \beta)
$$

Note that this transformation for the perturbative fields also depends on the homogeneous degrees of freedom. While the original perturbation variables had canonical momenta properly defined by the Legendre transform, the new perturbation variables will break the canonical structure as 
they non-trivially depend on the homogeneous degrees of freedom. In order to preserve the canonical structure of the system, it is mandatory to find a suitable transformation for the homogeneous and isotropic variables, too. This appears to be a cumbersome mission. Castelló Gomar et al. (2015) have however shown that it is possible to find a transformation for the homogeneous and isotropic degrees of freedom which preserves the canonical structure of the system up to second order in the cosmological scalar perturbations. The same has been done by Martínez and Olmedo (2016) for the tensor degrees of freedom.

These transformations and the resulting almost-canonical variables in the homogeneous and the perturbative sector lead to a redefinition of the Hamilton constraint. Fortunately, these transformations make the Dirac constraint analysis almost trivial. The secondary constraints that one obtains from requiring the conservation of the primary constraints under the evolution of the Hamiltonian, generate only one single, non-trivial constraint, namely,

$$
\mathcal{C}:=\mathcal{H}_{0}+\tilde{\mathcal{H}}_{2}^{s}+\check{\mathcal{H}}_{2}^{t}=0,
$$

where $\mathcal{H}_{0}$ now depends on the transformed homogeneous variables, and $\tilde{\mathcal{H}}_{2}^{s}$ and $\check{\mathcal{H}}_{2}^{t}$ are second order constraints that depend on the Mukhanov-Sasaki and the tensor perturbations respectively, as well as on the transformed homogeneous variables. Before we give the corresponding expressions, let us perform the typical $\varepsilon$-rescalings of the momenta that will make the space adiabatic scheme work at the technical level. We will simply denote the transformed variables by the original ones, and write,

$$
p_{a}:=\varepsilon^{2} P_{a}, \quad \quad p_{\phi}:=\varepsilon P_{\phi} .
$$

Besides, we perform a canonical rescaling of the inhomogeneous Mukhanov-Sasaki and tensor perturbations, and multiply the constraint by a global $\varepsilon^{2}$-factor. Then, the total Hamilton constraint, $C=H_{0}+\tilde{H}_{2}^{s}+\breve{H}_{2}^{t}=0$, is given by (Schander and Thiemann, 2019c),

$$
\begin{gathered}
H_{0}=-\frac{p_{a}^{2}}{12 a}+\frac{p_{\phi}^{2}}{2 a^{3}}+\frac{1}{2} \varepsilon^{2} m_{\Phi}^{2} a^{3} \phi^{2}+\Lambda a^{3}, \\
\tilde{H}_{2}^{s}=\frac{1}{2 a} \int_{\mathbb{T}^{3}} \mathrm{~d} x\left[\frac{\pi_{\vartheta}^{2}}{\sqrt{{ }^{0} \tilde{h}}}+\vartheta \varepsilon^{4}\left[-\sqrt{{ }^{0} \tilde{h}} \Delta+m_{\mathrm{MS}}^{2}\right] \vartheta\right], \\
\widehat{H}_{2}^{t}=\frac{1}{2 a} \int_{\mathbb{T}^{3}} \mathrm{~d} x\left[\frac{\pi_{t}^{a b} \pi_{t, a b}}{6 \sqrt{{ }^{0} \tilde{h}}}+t^{a b} \varepsilon^{4}\left[-3 \sqrt{{ }^{0} \tilde{h}} \Delta+\left(\varepsilon m_{\mathrm{T}}\right)^{2}\right] t_{a b}\right],
\end{gathered}
$$

with the effective Mukhanov-Sasaki and tensor masses depending on the homogeneous variables,

$$
\begin{gathered}
m_{\mathrm{MS}}^{2}=\left(-\frac{p_{a}^{2}}{18 a^{2}}+\frac{7 p_{\phi}^{2}}{2 a^{4}}-12 \varepsilon{\sqrt{{ }^{0} \tilde{h}}}^{2} m_{\Phi}^{2} \frac{a \phi p_{\phi}}{p_{a}}-18 \frac{p_{\phi}^{4}}{a^{6} p_{a}^{2}}+{\sqrt{{ }^{0} \tilde{h}}}^{2} m_{\Phi}^{2} a^{2}\right) \frac{1}{\sqrt{{ }^{0} \tilde{h}}} \\
\left(\varepsilon m_{\mathrm{T}}\right)^{2}=\left(\frac{p_{a}^{2}}{6 a^{2}}-3 \varepsilon^{2} \sqrt{{ }^{0} \tilde{h}^{2}} m_{\Phi}^{2} a^{2} \phi^{2}-6{\sqrt{{ }^{0} \tilde{h}}}^{2} \Lambda a^{2}\right) \frac{1}{\sqrt{{ }^{0} \tilde{h}}}
\end{gathered}
$$

Now, SAPT requires to quantize the cosmological perturbations. The form of $\tilde{H}_{2}^{s}$ and $\check{H}_{2}^{t}$ suggests to consider a standard Fock quantization for the Mukhanov-Sasaki and the tensor fields. The quantized fields should satisfy the commutation relations,

$$
\begin{aligned}
{\left[g(\vartheta), \boldsymbol{\pi}_{\vartheta}(f)\right]_{\mathrm{MS}} } & =i \int_{\mathbb{T}^{3}} \mathrm{~d} x g(x) f(x) 1_{\mathrm{MS}},\left[G(\boldsymbol{t}), \boldsymbol{\pi}_{t}(F)\right]_{\mathrm{T}} \\
& =i \int_{\mathbb{T}^{3}} \mathrm{~d} x G^{a b}(x) F_{a b}(x) 1_{\mathrm{T}}
\end{aligned}
$$

for suitable test functions $f, g, G^{a b}$ and $F_{a b}$ on the three-torus, where $1_{\mathrm{MS}}$ and $1_{\mathrm{T}}$ denote the unities of the quantum algebras. As a basis for the one-particle Hilbert space $L^{2}\left(\mathbb{T}^{3}, \mathrm{~d} x\right)$ it is convenient to choose the plane waves $f_{\vec{k}}(\vec{x})=\exp (i \vec{k} \vec{x})$, with $\vec{k} \in 2 \pi \mathbb{Z} 0$ being discrete. The total perturbative Hilbert space is the tensor product of the correspondent Fock spaces,

$$
\mathcal{H}_{\mathrm{f}}=\mathcal{F}_{\mathrm{s}, \mathrm{MS}}\left(L^{2}\left(\mathbb{T}^{3}, \mathrm{~d} x\right)\right) \underset{\tau=\{+,-\}}{\otimes} \mathcal{F}_{\mathrm{s}, \mathrm{T}, \tau}\left(L^{2}\left(\mathbb{T}^{3}, \mathrm{~d} x\right)\right),
$$

where the index " $\mathrm{s}$ " refers to symmetric, and we introduced the label $\tau$ which stands for the only two physical degrees of freedom associated with the tensor perturbations (namely their polarizations). The form of the second order contributions to the Hamilton constraint suggest to define the one-particle frequency operators for the Mukhanov-Sasaki and the tensor systems,

$$
\omega_{\mathrm{MS}}:=\varepsilon^{2} \sqrt{-\Delta+m_{\mathrm{MS}}^{2}}, \quad \omega_{\mathrm{T}}:=\varepsilon^{2} \sqrt{-18 \Delta+6\left(\varepsilon m_{\mathrm{T}}\right)^{2}} .
$$

Note that both operators depend on the homogeneous degrees of freedom as they contain the mass functions $m_{\mathrm{MS}}\left(a, p_{a}, \phi, p_{\phi}\right)$ and $m_{\mathrm{T}}\left(a, p_{a}, \phi\right)$. This implies that the annihilation and creation operators $\boldsymbol{a}\left(f_{\vec{k}}\right)=: \underset{\vec{k}}{\vec{a}}, \boldsymbol{a}^{*}\left(f_{\vec{k}}\right)=: \boldsymbol{a}_{\vec{k}}^{*}$ of the Mukhanov-Sasaki system and the annilation and creation operators of the tensor perturbations $\boldsymbol{b}_{ \pm}\left(f_{\vec{k}}\right)=\boldsymbol{b}_{\vec{k}, \pm}, \boldsymbol{b}_{ \pm}^{*}\left(f_{\vec{k}}\right)=\boldsymbol{b}_{\vec{k}, \pm}^{*}$, defined in the standard way, depend on the homogeneous degrees of freedom, and so do the natural Fock basis states (Schander and Thiemann, 2019c). In contrast to the cosmological toy model, they depend on the whole set of phase space variables, which represent noncommuting operators in the quantum theory, and which makes the application of SAPT mandatory. The Born-Oppenheimer approach would fail in the given case.

It is most convenient to express the quantum constraint symbol $\boldsymbol{C}\left(a, p_{a}, \phi, p_{\phi}\right)$ in terms of normal-ordered annihilation and creation operators,

$$
\begin{aligned}
\boldsymbol{C}= & \left(-\frac{p_{a}^{2}}{12 a}+\frac{p_{\phi}^{2}}{2 a^{3}}+\frac{1}{2} \varepsilon^{2} m_{\Phi}^{2} a^{3} \phi^{2}+\Lambda a^{3}\right) 1_{\mathrm{f}} \\
& +\frac{1}{a} \sum_{\vec{k} \in \mathbb{K}} \omega_{\mathrm{MS}, \vec{k}} \boldsymbol{a}_{\vec{k}}^{*} \boldsymbol{a}_{\vec{k}}+\frac{1}{6 a} \sum_{\vec{K} \in \mathbb{K}} \omega_{\mathrm{T}, \vec{k}} \boldsymbol{b}_{\vec{K}}^{*} \boldsymbol{b}_{\vec{K}},
\end{aligned}
$$

where we identify the first contribution as the usual FLRW Hamilton constraint, which we will denote by $E_{\text {hom }}\left(a, p_{a}, \phi, p_{\phi}\right)$. The label $\vec{K}:=(\vec{k}, \tau) \in \mathbb{K}$ specifies the mode and the polarization of the tensor perturbations. $C$ admits a discrete spectrum for any point $\left(a, p_{a}, \phi, p_{\phi}\right) \in \Gamma_{\mathrm{s}}$ 
because the sums over the wave vectors in the Hamilton constraint are discrete and so is the spectrum of the number operators $\underset{k}{\boldsymbol{a}} \underset{k}{\boldsymbol{a}}$ and $\boldsymbol{b}_{\vec{K}}^{*} \boldsymbol{b}_{\vec{K}}$. Any Fock state $\xi_{(n)} \in \mathcal{H}_{\mathrm{f}}$ with finite energy can be identified with a finite set of non-vanishing quantum numbers $(n):=\left\{\ldots, n_{\mathrm{MS}, \vec{k}_{1}}, n_{\mathrm{MS}, \vec{k}_{2}}, \ldots, n_{\mathrm{T}, \tau, \vec{k}_{1}}, n_{\mathrm{T}, \tau}, \vec{k}_{2}, \ldots\right\}$, where we distinguished between the quantum numbers of the Mukhanov-Sasaki and the tensor perturbations. We introduce degeneracy labels which take the possibility of degenerate eigenstates into account, and we denote them by $b=1, \ldots, d$ for the Mukhanov-Sasaki system and $b^{\prime}=1, \ldots, d^{\prime}$ for the graviton system. To shorten notation, we integrate the degeneracy labels in $\beta:=\left\{b, b^{\prime}\right\}$ and the degeneracy numbers in $\delta:=\left\{d, d^{\prime}\right\}$. We write for the set of homogeneous variables, $(q, p):=\left(a, p_{a}, \phi, p_{\phi}\right)$. The eigenvalue problem for any finite set of quantum numbers $(n)_{\beta}$ then has the form,

$$
\begin{gathered}
C(q, p) \xi_{(n)_{\beta}}(q, p)=E_{n}(q, p) \xi_{(n)_{\beta}}(q, p), \\
E_{n}(q, p):=E_{\mathrm{hom}}\left(a, p_{a}, \phi, p_{\phi}\right)+\frac{1}{a} \sum_{\vec{k} \in \mathbb{K}} n_{\mathrm{MS}, \vec{k}, b} \omega_{\mathrm{MS}, \vec{k}} \\
+\frac{1}{6 a} \sum_{\vec{K} \in \mathbb{K}} n_{\mathrm{T}, \vec{K}, b^{\prime}} \omega_{\mathrm{T}, \vec{K}} .
\end{gathered}
$$

Due to the discreteness of the eigenbasis, it is possible to define non-vanishing energy gaps between the eigenenergy bands of the perturbations, at least for local regions in phase space. In the following, we assume that the relevant energy bands admit such local gaps in the region of interest.

To examine the derivatives of the eigenstates with respect to the homogeneous variables, we need the derivative of the vacuum state and the annihilation operators since any excited state in the Hilbert space $\mathcal{H}_{\mathrm{f}}$ can be constructed from the vacuum state $\Omega(q, p)$ by applying the desired number $\left(n_{\mathrm{MS}, \vec{k}}, n_{\mathrm{T}, \vec{k},+}, n_{\mathrm{T}, \vec{k},-}\right)$ of creation operators for every wave number $\vec{k}$. We formally choose one such eigenstate with quantum number $(s)(\nu)_{\beta}$ given by,

$$
\xi_{(\nu)}(q, p)=\prod_{\vec{K} \in \mathbb{K}} \frac{\left(\boldsymbol{a}_{\vec{k}}^{*}\right)^{\nu, \overrightarrow{M S}}}{\sqrt{\nu_{\mathrm{MS}, \vec{k}} !}} \frac{\left(\boldsymbol{b}_{\vec{K}}^{*}\right)^{\nu_{\mathrm{T}}, \vec{K}}}{\sqrt{\nu_{\mathrm{T}, \vec{K}} !}} \Omega(q, p)
$$

It is useful to write the explicit representation of the Mukhanov-Sasaki wave function and the tensor wave functions as a product,

$$
\xi_{(\nu)}=: \xi_{\left(\nu_{\mathrm{MS}}\right)}^{\mathrm{MS}} \cdot \prod_{\tau} \xi_{\left(\nu_{\mathrm{T}}\right)}^{\mathrm{T}, \tau}
$$

The derivatives of the annihilation operators with respect to $\lambda \in\{q, p\}$, are proportional to the correspondent creation operators,

$$
\begin{aligned}
& \frac{\partial \boldsymbol{a}_{\vec{k}}(q, p)}{\partial \lambda}:=f_{\lambda, \vec{k}}^{\mathrm{MS}}(q, p) \boldsymbol{a}_{\vec{k}}^{*}(q, p), \\
& \frac{\partial \boldsymbol{b}_{\vec{K}}(q, p)}{\partial \lambda}:=f_{\lambda, \vec{K}}^{\mathrm{T}}(q, p) \boldsymbol{b}_{\vec{K}}^{*}(q, p),
\end{aligned}
$$

and the explicit expressions of the factors can be found in (Schander and Thiemann, 2019c). The $\lambda$-derivative of the vacuum state is then given by,

$$
\begin{aligned}
\frac{\partial \Omega(q, p)}{\partial \lambda}= & \sum_{\vec{k} \in \mathbb{K}} f_{\lambda, \vec{k}}^{\mathrm{MS}}(q, p)\left(\underset{\vec{k}}{\boldsymbol{a}_{\vec{k}}^{*}} \boldsymbol{a}_{\vec{k}}^{*} \Omega\right)(q, p) \\
& +\sum_{\vec{K} \in \mathbb{K}} f_{\lambda \vec{k}}^{\mathrm{T}}(q, p)\left(\boldsymbol{b}_{\vec{K}}^{*} \boldsymbol{b}_{\vec{K}}^{*} \Omega\right)(q, p) .
\end{aligned}
$$

The $\lambda$-derivative of any excited state $\xi_{(n)}$ is thus uniquely defined by these results and can be expressed as an application of a connection $\mathcal{A}_{\lambda} \in C^{\infty}\left(\Gamma_{\mathrm{s}}, \mathcal{L}\left(\mathcal{H}_{\mathrm{f}}\right)\right)$, on the global Hilbert bundle $H$,

$$
\begin{aligned}
& \frac{\partial \xi_{(n)}(q, p)}{\partial \lambda}=: \mathcal{A}_{\lambda} \xi_{(n)}=: \mathcal{A}_{\lambda(n)}^{(m)} \xi_{(m)}, \\
& \mathcal{A}_{\lambda(n)}^{(m)}(q, p) \in C^{\infty}\left(\Gamma_{s}, \mathbb{R}\right) \quad \forall(n),(m),
\end{aligned}
$$

where the summation over $(m)$ includes essentially all possible excitation numbers within the Fock space $\mathcal{H}_{\mathrm{f}}$. However, there is only a countable number of $(m)$ 's for which $\mathcal{A}_{\lambda(n)}^{(m)}$ is non-vanishing if $(n)$ is a finite set of non-vanishing excitation numbers. For more details and the explicit calculations, we refer to (Schander and Thiemann, 2019c).

\section{Application of a Space Adiabatic Perturbation Scheme}

The construction of the space adiabatic symbols is subject to two different perturbative scalings: $\varepsilon$ for the homogeneous scalar field, and $\varepsilon^{2}$ for the homogeneous gravitational degrees of freedom. The Moyal product for two operator-valued functions $\boldsymbol{f}(q, p) \in S_{\rho}^{m_{1}}\left(\Gamma_{\mathrm{s}}, \mathcal{B}\left(\mathcal{H}_{\mathrm{f}}\right)\right), \boldsymbol{g}(q, p) \in S_{\rho}^{m_{2}}\left(\Gamma_{\mathrm{s}}, \mathcal{B}\left(\mathcal{H}_{\mathrm{f}}\right)\right)$ has the form,

$$
\begin{aligned}
\left(\boldsymbol{f} \star_{\varepsilon} \boldsymbol{g}\right)(q, p) \asymp\left[\boldsymbol { f } \operatorname { e x p } \left(\frac{i \varepsilon}{2}\left(\overleftarrow{\partial}_{\phi} \vec{\partial}_{p_{\phi}}-\overleftarrow{\partial}_{p_{\phi}} \vec{\partial}_{\phi}\right)\right.\right. \\
\left.\left.-\frac{i \varepsilon^{2}}{2}\left(\overleftarrow{\partial}_{a} \vec{\partial}_{p_{a}}-\overleftarrow{\partial}_{p_{a}} \vec{\partial}_{a}\right)\right) \boldsymbol{g}\right](q, p) \in S_{\rho}^{m_{1}+m_{2}}\left(\Gamma_{s}, \mathcal{B}\left(\mathcal{H}_{\mathrm{f}}\right)\right)
\end{aligned}
$$

As it turns out, the Moyal product with respect to the gravitational degrees of freedom does not contribute to the computations up to second order in the perturbation scheme.

As before, the discrete eigenstate $\xi_{(\nu)_{\beta}}(q, p) \in \mathcal{H}_{\mathrm{f}}$ with quantum number $(\nu)_{\beta}$ serves to define the zeroth order Moyal projector symbol,

$$
\pi_{0}(q, p):=\sum_{\beta} \xi_{(v)_{\beta}}(q, p)\left\langle\xi_{(\nu)_{\beta}}(q, p), \cdot\right\rangle_{\mathrm{f}}
$$

The only relevant contribution to $\pi_{1}$ comes from (S1-3). This off-diagonal part $\pi_{1}^{\mathrm{OD}}$ mixes the adjacent inhomogeneous eigenstates according to,

$$
\pi_{1}^{\mathrm{OD}}=\frac{i}{2} \sum_{\beta=1}^{\delta} \sum_{(n) \neq(v)_{\beta}} \frac{A_{(v)_{\beta}(n)}}{E_{(v)_{\beta}}-E_{(n)}}\left(\xi_{(v)_{\beta}}\left\langle\xi_{(n)}, \cdot\right\rangle_{\mathrm{f}}-\xi_{(n)}\left\langle\xi_{(v)_{\beta}}, \cdot\right\rangle_{\mathrm{f}}\right),
$$

with 


$$
\begin{aligned}
A_{(\nu)_{\beta}(n)}= & \mathcal{A}_{\phi(\nu)_{\beta}} \frac{\partial\left(E_{(n)}+E_{(\nu)_{\beta}}\right)}{\partial p_{\phi}}-\mathcal{A}_{p_{\phi}(v) \beta} \frac{\partial\left(E_{(n)}+E_{(v)_{\beta}}\right)}{\partial \phi} \\
& +\left(E_{(n)}-E_{(m)}\right)\left(\mathcal{A}_{\phi_{\phi}(v)}^{(m)} \mathcal{A}_{\phi(m)}^{(n)}-\mathcal{A}_{\phi(\nu)_{\beta}}^{(m)} \mathcal{A}_{p_{\phi}(m)}^{(n)}\right) .
\end{aligned}
$$

Constructing the unitary symbol $\boldsymbol{\pi}_{(1)}$ requires to choose a simple reference space $\mathcal{K}_{\mathrm{f}}$, and as before, $\mathcal{H}_{\mathrm{f}}$ itself is a convenient choice. Its basis is determined by fixing a set of numbers $\left(q_{0}, p_{0}\right) \in \Gamma_{\mathrm{s}}$ giving $\left\{\zeta_{(n)}:=\xi_{(n)}\left(q_{0}, p_{0}\right)\right\}_{(n)}$. The zeroth order contribution to the Moyal unitary and the reference projector can be chosen as,

$$
\boldsymbol{u}_{0}(q, p):=\sum_{(n)} \zeta_{(n)}\left\langle\xi_{(n)}(q, p), \cdot\right\rangle_{\mathrm{f}}, \quad \boldsymbol{\pi}_{\mathrm{R}}:=\sum_{\beta=1}^{\delta} \zeta_{(\nu)_{\beta}}\left\langle\zeta_{(\nu)}, \cdot\right\rangle_{\mathrm{f}} .
$$

The hermitian part of $\boldsymbol{u}_{1}(q, p)$ is determined by evaluating (S2-1) and (S2-2) up to first order in the perturbations,

$$
\begin{aligned}
\boldsymbol{u}_{1}^{\mathrm{h}}(q, p)= & \sum_{(n),(m),(k)}\left(\mathcal{A}_{\phi(n)}^{(m)} \mathcal{A}_{p_{\phi}(m)}^{(k)}\right. \\
& \left.-\mathcal{A}_{p_{\phi}(n)}^{(m)} \mathcal{A}_{\phi(m)}{ }^{(k)}\right) \zeta_{(n)}\left\langle\zeta_{(k)}, \cdot\right\rangle_{\mathrm{f}} .
\end{aligned}
$$

Since the sum runs over all possible combinations of quantum numbers, it is clear that the two contributions are equal and cancel each other. We thus have that $\boldsymbol{u}_{1}^{\mathrm{h}}=0$. The antihermitian part of $\boldsymbol{u}_{1}$ results from employing the result for $\pi_{1}^{\mathrm{OD}}$ in the well-known expression from the toy model example,

$$
\boldsymbol{u}_{1}^{\mathrm{ah}}=\left[\boldsymbol{\pi}_{\mathrm{R}}, \boldsymbol{u}_{0} \cdot \boldsymbol{\pi}_{1}^{\mathrm{OD}} \cdot \boldsymbol{u}_{0}{ }_{\mathrm{f}} \cdot \boldsymbol{u}_{0} .\right.
$$

We evaluate the effective Hamilton constraint symbol according to, $C_{\text {eff }}=\boldsymbol{u} \star_{\varepsilon} \boldsymbol{C} \star_{\varepsilon} \boldsymbol{u}^{\star}$, and restrict our interest directly to the reference space, i.e., to $C_{\text {eff, } R}=\pi_{R} \cdot C_{\text {eff }} \cdot \pi_{R}$. At zeroth order, this yields,

$$
\begin{aligned}
& C_{\mathrm{eff}, 0, \mathrm{R}}=\sum_{b, b^{\prime}=1}^{d, d^{\prime}}\left[E_{\mathrm{hom}}\left(a, p_{a}, \phi, p_{\phi}\right)+\frac{1}{a} \sum_{\vec{k} \in \mathbb{K}} v_{\mathrm{MS}, \vec{k}, b} \omega_{\mathrm{MS}, \vec{k}}\right. \\
& \left.+\frac{1}{6 a} \sum_{\vec{K} \in \mathbb{K}} v_{\mathrm{T}, \vec{K}, b^{\prime}} \omega_{\mathrm{T}, \vec{K}}\right] \cdot \zeta_{(v)_{\beta}}\left\langle\zeta_{\left.()_{\beta}\right)^{\prime}},\right\rangle_{\mathrm{f}},
\end{aligned}
$$

which includes the standard zeroth order Hamilton constraint for an FLRW Universe $E_{\mathrm{hom}}\left(a, p_{a}, \phi, p_{\phi}\right)$, and the bare energy contributions from the relevant energy band $\xi_{(v)_{a}}$. These additional terms are finite since the quantum numbers $\left\{v_{\mathrm{MS}, \vec{k}, b}, v_{\mathrm{T}, \vec{k}, b^{\prime}}\right\}$ are non-vanishing for only a finite number of wave vectors $\vec{k}$. Te first order contribution to the effective Hamiltonian vanishes identically within the subspace of interest.

The second order effective Hamilton symbol includes several contributions but only one of them is of second order in the perturbative parameter, and hence relevant.
The occurence of terms that actually enter at higher orders in $\varepsilon$ stems from the fact that the perturbative Mukhanov-Sasaki and graviton contributions to $C$ are by definition of second order in $\varepsilon$. It was necessary to include them to make the space adiabatic scheme work at the technical level. We refer again to (Schander and Thiemann 2019c) for more details and only state the final result,

$$
\begin{aligned}
C_{\text {eff }, 2, \mathrm{R}}\left(a, p_{a}, \phi, p_{\phi}\right)= & -\sum_{b=1}^{d} \sum_{\vec{k} \in \mathbb{K}} \frac{1}{\left(\vec{k}^{2}+m_{\mathrm{MS}}^{2}\right)^{5 / 2}}\left(\nu_{\mathrm{MS}, \vec{k}, b}+\frac{1}{2}\right) \\
& \frac{9}{2} \frac{m_{\Phi}^{4} p_{\phi}^{4}}{a^{3} p_{a}^{2}} \zeta_{\left(v_{b}\right)_{b}}\left\langle\zeta_{\left(v_{b}\right)},\right\rangle_{\mathrm{f}} .
\end{aligned}
$$

This second order effective Hamiltonian symbol together with the zeroth order contribution Eq. 80, provides after Weyl quantization a constraint operator for the homogeneous sector of quantum gravity which includes, most importantly, the backreaction from the inhomogeneous modes. A similar result was obtained for a quantum cosmological model with scalar field perturbations and a deparametrizing dust particle (Schander and Thiemann, 2019b). The next step of the scheme consequently consists in Weyl quantizing the full effective constraint symbol and in finding physical quantum states on the homogeneous Hilbert space that are annihilated by it. A thorough discussion of the above results will be given in the next and final section.

\section{DISCUSSION AND OUTLOOK}

This review provides an introduction to the backreaction problem in classical, semiclassical and quantum cosmology, as well as a detailed overview of the current state of research in the respective fields. We have particularly focused on approaches to the backreaction problem in (perturbative) quantum cosmology that are inspired by Born-Oppenheimer methods. The main part of this paper is dedicated to a program which uses SAPT as due to Panati et al. (2003), and which extends the latter scheme to quantum field theoretical models. Thereby, it is possible to compute the backreaction effects from the quantum cosmological perturbations on the homogeneous and isotropic quantum background (Schander and Thiemann, 2019b; Schander and Thiemann, 2019c). We have advocated this framework here as it represents an unambigious and straighforward formalism in order to incorporate the yet neglected backreaction effects in quantum cosmology in a perturbative and rigorous way.

The extension of the SAPT methods actually requires some care. The first issue is related to a violation of the Hilbert-Schmidt condition in QFT on CST. In fact, it is well-known from standard QFT that Klein-Gordon fields with different masses give rise to unitarily inequivalent representations of the field algebra (Haag, 1992). Since here, the effective masses of the Klein-Gordon and tensor fields depend on the homogeneous FLRW background, the theory prevents unitarily equivalent quantum field theories for different background configurations. 
This would evidently impede the quantization of the homogeneous sector. Schander and Thiemann (2019a,c) show that it is possible to circumvent these problems by considering transformations of the whole system that are canonical up to second order in the cosmological perturbations (Castelló Gomar et al., 2015; Martínez and Olmedo, 2016; Schander and Thiemann, 2019b).

Another point is that the mass squared functions of the perturbative quantum fields $m_{\mathrm{MS}}^{2}\left(a, p_{a}, \phi, p_{\phi}\right)$ and $\left(\varepsilon m_{\mathrm{T}}\right)^{2}\left(a, p_{a}, \phi\right)$ in Eqs. 62 and $\mathbf{6 3}$ are indefinite, and which leads to tachyonic instabilities. In (Schander and Thiemann, 2019a), several solutions are propsed, for example to revise the almost-canonical transformations that have actually led to these indefinite mass functions. A second proposal is to restrict the homogeneous phase space of the theory to regions in which the mass functions are positive. This can be made manifest by performing coordinate transformations in the slow sector. This is exemplified for the model with gauge-invariant perturbations in (Radzikowski, 2008; Schander and Thiemann, 2019a).

With the identification and solutions to these initial problems, it was possible to successfully apply the methods of SAPT to the backreaction problem in quantum cosmology. We stated the results for a cosmological, homogeneous and isotropic toy model, and for a fully-fledged perturbative quantum cosmology with gauge-invariant perturbations (Schander and Thiemann, 2019c; Neuser et al., 2019). In the first case, this effective Hamilton constraint includes the backreaction of the homogeneous scalar field; in the second case, the backreactions of the perturbative degrees of freedom on the homogeneous background are taken into account. Here, results up to second order in the adiabatic $\mathcal{E}$-scheme are presented. The effective Hamiltonian symbol eventually needs to be quantized with respect to the slow sector and the goal is to find admissible solutions. This has been done for an oscillator toy model in (Neuser et al., 2019). One can proceed here in the same way but analytic solutions are harder to find due to the non-polynomial structure of the result, and which requires an in depth analysis of their dense domain. For simplicity, we chose a Weyl quantization scheme and a Schrödinger representation following the original work by Panati et al. (2003). Instead, one could consider the representation underlying LQC which could be of advantage regarding the domain issues (Bojowald, 2008). Due to the peculiarities of that representation (especially the strong discontinuity of the Weyl elements), and in agreement with certain superselection structures of the dynamics, one would need to discretize the labels of the Weyl elements in one of the conjugate variables. This would effectively replace the gravitational slow phase space $T^{*} \mathbb{R}$ by $T^{*}\left(S^{1}\right)$ for which the Weyl quantization in application to LQC has been discussed in (Stottmeister and Thiemann, 2016b).

Focusing on the second order contribution to the perturbative model, Eq. 81, one might be worried about the infinite sums.
Note that the result splits into two parts, namely the one including the finite number of non-vanishing relevant quantum numbers $v_{\mathrm{MS}, \vec{k}, b}$ for different degeneracy labels $b$, and the contributions which do not depend on these quantum numbers and hence include any summand of the wave vector sum. The first part has only a finite number of contributions and is manageable, while the second includes in principle an infinite sum. Fortunately, the wave vector square enters with an exponent of $-5 / 2$ which makes the sum a priori a convergent sum. But the effective Mukhanov-Sasaki mass squared $m_{\mathrm{MS}}^{2}\left(a, p_{a}, \phi, p_{\phi}\right)$ in the denominator is an indefinite function on the homogeneous phase space. This will be cured as soon as a positive definite sector of the mass squared functions has been found.

We emphasize again that the issue of convergence of the perturbation series in the SAPT approach has not been addressed here. We point to easily implementable strategies (Panati et al., 2003; Stottmeister, 2015), that allow to define auxiliary Hamiltonian symbols that capture the relevant physics of the model under consideration and whose perturbation series is safely convergent.

Finally, we stress that there is an obvious connection between backreaction and decoherence (Schlosshauer, 2007). Indeed, in decoherence, one aims at finding an effective description of what we call the slow sector using the reduced density matrix approach, tracing over the fast degrees of freedom (Kiefer, 1987; Paz and Sinha, 1991, 1992) (and references therein), and computing its effective dynamics, e.g., by solving associated Lindblad equations (Manzano, 2020). Using the tensor product structure of the full Hilbert space, the connection to our approach would be to construct the reduced density matrix from a density matrix on the full Hilbert space that can be formed from the eigenstates of the Hamiltonian (constraint) corresponding to a given energy band. Details will be given elsewhere.

\section{AUTHOR CONTRIBUTIONS}

SS and TT both contributed to all aspects of the present review.

\section{ACKNOWLEDGMENTS}

We thank Kasia Rejzner for helpful references and information on the backreaction problem within the algebraic approach to QFT. The work of SS is supported by Perimeter Institute for Theoretical Physics. Research at Perimeter Institute is supported in part by the Government of Canada through the Department of Innovation, Science and Economic Development and by the Province of Ontario through the Ministry of Colleges and Universities. 


\section{REFERENCES}

Abramo, L. R. W., Brandenberger, R. H., and Mukhanov, V. F. (1997). Energymomentum Tensor for Cosmological Perturbations. Phys. Rev. D 56, 3248-3257. arXiv: gr-qc/9704037. doi:10.1103/physrevd.56.3248

Abramo, L. R., and Woodard, R. P. (2002). No One Loop Back Reaction in Chaotic Inflation. Phys. Rev. D 65, 063515. arXiv: astro-ph/0109272. doi:10.1103/ PhysRevD.65.063515

Adamek, J., Clarkson, C., Daverio, D., Durrer, R., and Kunz, M. (2019). Safely Smoothing Spacetime: Backreaction in Relativistic Cosmological Simulations. Class. Quan. Grav. 36, 014001. arXiv: 1706.09309 [astro-ph.CO]. doi:10.1088/ 1361-6382/aaeca5

Adamek, J., Daverio, D., Durrer, R., and Kunz, M. (2016). Gevolution: a Cosmological N-Body Code Based on General Relativity. J. Cosmol. Astropart. Phys. 2016, 053. arXiv: 1604.06065 [astro-ph.CO]. doi:10.1088/ 1475-7516/2016/07/053

Ade, P. A. R., Aghanim, N., Arnaud, M., Ashdown, M., Aumont, J., Baccigalupi, C., et al. (2016). Planck 2015 Results. XIV. Dark Energy and Modified Gravity. Astron. Astrophys. 594, À14. arXiv: 1502.01590 [astro-ph.CO].

Aghanim, N., Arroja, F., Ashdown, M., Aumont, J., Baccigalupi, C., Ballardini, M., et al. (2019). Planck 2018 Results. I. Overview and the Cosmological Legacy of Planck. Available at: arXiv: 1807.06205 [astro-ph.CO].

Aghanim, N., Akrami, Y., Ashdown, M., Aumont, J., Baccigalupi, C., Ballardini, M., et al. (2020). Planck 2018 Results. VI. Cosmological Parameters. Available at: arXiv: 1807.06209 [astro-ph.CO].

Agullo, I., Ashtekar, A., and Nelson, W. (2013). Extension of the Quantum Theory of Cosmological Perturbations to the Planck Era. Phys. Rev. D 87 (4), 043507. arXiv: 1211.1354 [gr-qc]. doi:10.1103/physrevd.87.043507

Alpher, R. A., and Herman, R. (1948a). Evolution of the Universe. Nature 162, 774-775. doi:10.1038/162774b0

Alpher, R. A., and Herman, R. C. (1948b). On the Relative Abundance Of the Elements. Phys. Rev. 74, 1737-1742. doi:10.1103/physrev.74.1737

Anderson, P. (1983). Effects of Quantum fields on Singularities and Particle Horizons in the Early Universe. Phys. Rev. D 28, 271-285. doi:10.1103/ physrevd.28.271

Anderson, P. R. (1984). Effects of Quantum fields on Singularities and Particle Horizons in the Early Universe. II. Phys. Rev. D 29, 615-627. doi:10.1103/ physrevd.29.615

Anderson, P. R. (1985). Effects of Quantum fields on Singularities and Particle Horizons in the Early Universe. III. The Conformally Coupled Massive Scalar Field. Phys. Rev. D 32, 1302-1315. doi:10.1103/physrevd.32.1302

Araki, H. (1999). Mathematical Theory of Quantum fields. New York: Oxford University Press.

Ashtekar, A., Bojowald, M., and Lewandowski, J. (2003). Mathematical Structure of Loop Quantum Cosmology. Adv. Theor. Math. Phys. 7, 233-268. arXiv: gr-qc/ 0304074. doi:10.4310/atmp.2003.v7.n2.a2

Barausse, E., Matarrese, S., and Riotto, A. (2005). Effect of Inhomogeneities on the Luminosity Distance-Redshift Relation: Is Dark Energy Necessary in a Perturbed Universe?. Phys. Rev. D 71, 063537, 2005 . arXiv: astro-ph/ 0501152. doi:10.1103/PhysRevD.71.063537

Barausse, E., Matarrese, S., and Riotto, A. (2005). The Effect Of Inhomogeneities on the Luminosity Distance-Redshift Relation: Is Dark Energy Necessary in a Perturbed Universe? Phys. Rev. D 71, 063537. arXiv: astro-ph/0501152. doi:10.1103/physrevd.71.063537

Barrera-Hinojosa, C., and Li, B. (2020). GRAMSES: a New Route to General Relativistic N-Body Simulations in Cosmology. Part I. Methodology and Code Description. J. Cosmol. Astropart. Phys. 2020, 007. arXiv: 1905.08890 [astroph.CO]. doi:10.1088/1475-7516/2020/01/007

Baumann, D., Nicolis, A., Senatore, L., and Zaldarriaga, M. (2012). Cosmological Non-Linearities as an Effective Fluid. J. Cosmol. Astropart. Phys. 2012, 051. arXiv:1004.2488 [astro-ph.CO]. doi:10.1088/1475-7516/2012/07/051

Behrend, J., Brown, I. A., and Robbers, G. (2008). Cosmological Backreaction from Perturbations. J. Cosmol. Astropart. Phys. 2008, 013, 2008. arXiv: 0710.4964 [astro-ph]. doi:10.1088/1475-7516/2008/01/013

Berry, I. V. (1984). Quantal Phase Factors Accompanying Adiabatic Changes. Proc. R. Soc. Lond. A. 392, 45-57. doi:10.1098/rspa.1984.0023
Bertoni, C., Finelli, F., and Venturi, G. (1996). The Born - Oppenheimer Approach to the Matter - Gravity System and Unitarity. Class. Quan. Grav. 13, 2375-2383. arXiv: gr-qc/9604011. doi:10.1088/0264-9381/13/9/005

Bianchi, E., Rovelli, C., and Vidotto, F. (2010). Towards Spinfoam Cosmology. Phys. Rev. D 82, 084035. arXiv:1003.3483 [gr-qc]. doi:10.1103/ physrevd.82.084035

Birrell, N. D., and Davies, P. C. W. (1984). “Quantum Fields in Curved Space," in Cambridge Monographs on Mathematical Physics. Cambridge, UK: Cambridge Univ. Press.

Biswas, T., and Notari, A. (2008). 'Swiss-cheese' Inhomogeneous Cosmology and the Dark Energy Problem. J. Cosmol. Astropart. Phys. 2008, 021, 2008. arXiv: astro-ph/0702555. doi:10.1088/1475-7516/2008/06/021

Blaszak, M., and Domanski, Z. (2012). Phase Space Quantum Mechanics. Ann. Phys. 327, 167-211. doi:10.1016/j.aop.2011.09.006

Blumenthal, G. R., Faber, S. M., Primack, J. R., and Rees, M. J. (1984). "Formation of Galaxies and Large-Scale Structure with Cold Dark Matter," in Nature 311. Editor M. A. Srednicki, pp. 517-525. doi:10.1038/311517a0

Bojowald, M. (2008). Loop Quantum Cosmology. Living Rev. Relativ. 11, 4. doi:10.12942/lrr-2008-4

Bolejko, K., and Célérier, M.-N. (2010). Szekeres Swiss-cheese Model and Supernova Observations. Phys. Rev. D 82, 103510. arXiv: 1005.2584 [astroph.CO]. doi:10.1103/physrevd.82.103510

Bolejko, K, and Korzyński, M. (2017). Inhomogeneous Cosmology and Backreaction: Current Status and Future Prospects. Fourteenth Marcel Grossmann Meet. 14, (1), 602-621. arXiv: 1612.08222 [gr-qc]. doi:10.1142/ 97898132266090033

Bolejko, K., and Korzyński, M. (2017). "Inhomogeneous Cosmology and Backreaction: Current Status and Future Prospects, Int. J. Mod. Phys. D, 26". In: Int. J. Mod. Phys. D 26.06. Ed. by M. Bianchi, R. T. Jantzen, and R. Ruffini, p. 1730011, 2017 . arXiv: 1612.08222 [gr-qc]. doi:10.1142/ S0218271817300117

Bonvin, C., Durrer, R., and Gasparini, M. A. (2006). "Erratum: Fluctuations of the Luminosity Distance [Phys. Rev. D73, 023523 (2006)]". In: [Erratum: Phys. Rev. D 85,029901, 2006 (2012)], p. 023523. arXiv: astro-ph/0511183. doi:10.1103/ PhysRevD.85.029901

Brandenberger, R., Graef, L. L., Marozzi, G., and Vacca, G. P. (2018). Backreaction of Super-hubble Cosmological Perturbations beyond Perturbation Theory. Phys. Rev. D 98, 103523. arXiv: 1807.07494. doi:10.1103/PhysRevD.98.103523

Bonvin, C., Durrer, R., and Gasparini, M. (2006). Fluctuations of the Luminosity Distance. Phys. Rev. D 73, 023523. arXiv: astro-ph/0511183. doi:10.1103/ physrevd.73.023523

Born, M., and Oppenheimer, R. (1927). Zur Quantentheorie der Molekeln. Ann. Phys. 389, 457-484. doi:10.1002/andp.19273892002

Boyanovsky, D., D’Attanasio, M., de Vega, H. J., Holman, R., and Lee, D.-S. (1995) Linear versus Nonlinear Relaxation: Consequences for Reheating and Thermalization. Phys. Rev. D 52, 6805-6827. arXiv: hep-ph/9507414. doi:10.1103/physrevd.52.6805

Brandenberger, R., Graef, L. L., Marozzi, G., and Vacca, G. P. (2018). Backreaction of Super-Hubble Cosmological Perturbations beyond Perturbation Theory. Phys. Rev. D 98, 103523. arXiv: 1807.07494 [hep-th]. doi:10.1103/ physrevd.98.103523

Brandenberger, R. H., Feldman, H., and Mukhanov, V. F. (1993). "Classical and Quantum Theory of Perturbations in Inflationary Universe Models," in 37th Yamada Conference: Evolution of the Universe and its Observational Quest. Available at: arXiv: astro-ph/9307016.

Brandenberger, R. H. (2004). "Lectures on the Theory of Cosmological Perturbations," in Lecture Notes in Physics, The Early Universe and Observational Cosmology 646. Editors N. Breton, J. L. Cervantes-Cota, and M. Salgado, pp. 127-167. arXiv: hep-th/0306071. doi:10.1007/978-3-54040918-2_5

Briggs, J. S., and Rost, J. M. (2000). Time Dependence in Quantum Mechanics. Eur. Phys. J. D 10, 311. arXiv: quant-ph/9902035. doi:10.1007/s100530050554

Brizuela, D., and Krämer, M. (2018). Quantum-Gravitational Effects on Primordial Power Spectra in Slow-Roll Inflationary Models. Galaxies 6 (1), 6. doi:10.3390/ galaxies6010006

Brout, R. (1987). On the Concept Of Time and the Origin Of the Cosmological Temperature. Found. Phys. 17 (6), 603-619. doi:10.1007/bf01882790 
Brout, R., and Venturi, G. (1989). Time in Semiclassical Gravity. Phys. Rev. D 39, 2436-2439. doi:10.1103/physrevd.39.2436

Brown, I. A., Robbers, G., and Behrend, J. (2009). Averaging Robertson-Walker Cosmologies. J. Cosmol. Astropart. Phys. 2009, 016. arXiv: 0811.4495 [gr-qc]. doi:10.1088/1475-7516/2009/04/016

Brunetti, R., and Fredenhagen, K. (2000). Microlocal Analysis and Interacting Quantum Field Theories: Renormalization on Physical Backgrounds. Commun. Math. Phys. 208, 623-661. arXiv: math- ph/9903028. doi:10.1007/s002200050004

Buchert, T. (2008). Dark Energy from Structure: A Status Report. Gen. Relativ Gravit. 40, 467-527. arXiv: 0707.2153 [gr-qc]. doi:10.1007/s10714-007-0554-8

Buchert, T. (2000). On Average Properties of Inhomogeneous Fluids in General Relativity: Dust Cosmologies. Gen. Relativ. Gravitation 32, 105-125. arXiv: grqc/9906015. doi:10.1023/a:1001800617177

Buchert, T. (2001). On Average Properties of Inhomogeneous Fluids in General Relativity: Perfect Fluid Cosmologies. Gen. Relativ. Gravitation 33, 1381-1405. arXiv: gr-qc/0102049. doi:10.1023/a:1012061725841

Buchert, T., and Räsänen, S. (2012). Backreaction in Late-Time Cosmology. Annu. Rev. Nucl. Part. Sci. 62, 57-79. arXiv: 1112.5335 [astro-ph.CO]. doi:10.1146/ annurev.nucl.012809.104435

Cailleteau, T., Mielczarek, J., Barrau, A., and Grain, J. (2012). Anomaly-free Scalar Perturbations with Holonomy Corrections in Loop Quantum Cosmology. Class. Quan. Grav. 29, 095010. arXiv: 1111.3535 [gr-qc]. doi:10.1088/02649381/29/9/095010

Calzetta, E., and Hu, B. L. (1987). Closed-time-path Functional Formalism in Curved Spacetime: Application to Cosmological Back-Reaction Problems. Phys. Rev. D 35, 495-509. doi:10.1103/physrevd.35.495

Calzetta, E., and Hu, B. L. (1989). Dissipation of Quantum fields from Particle Creation. Phys. Rev. D 40, 656-659. doi:10.1103/physrevd.40.656

Calzetta, E., and Hu, B. L. (1994). Noise and Fluctuations in Semiclassical Gravity. Phys. Rev. D 49, 6636-6655. arXiv: gr-qc/9312036. doi:10.1103/physrevd.49.6636

Calzetta, E., and Hu, B. L. (1995). Quantum Fluctuations, Decoherence of the Mean Field, and Structure Formation in the Early Universe. Phys. Rev. D 52, 6770-6788. arXiv: gr-qc/9505046. doi:10.1103/physrevd.52.6770

Campos, A., and Verdaguer, E. (1994). Semiclassical Equations for Weakly Inhomogeneous Cosmologies. Phys. Rev. D 49, 1861-1880. arXiv: gr-qc/ 9307027. doi:10.1103/physrevd.49.1861

Castelló Gomar, L., Martín-Benito, M., and Mena Marugán, G. A. (2015). Gaugeinvariant Perturbations in Hybrid Quantum Cosmology. J. Cosmol. Astropart. Phys. 2015, 045, 2015. arXiv: 1503.03907 [gr-qc]. doi:10.1088/1475-7516/2015/ $06 / 045$

Castelló Gomar, L., Mena Marugán, G. A., and Martín-Benito, M. (2016). Quantum Corrections to the Mukhanov-Sasaki Equations. Phys. Rev. D 93, 2016, 104025. arXiv:1603.08448 [gr-qc]. doi:10.1103/physrevd.93.104025

Cervantes-Cota, J. L., and Smoot, G. (2011). Cosmology Today - A Brief Review. AIP Conf. Proc. 1396 (1), 28-52. arXiv: 1107.1789 [astro-ph.CO].

Clarkson, C., Ellis, G., Larena, J., and Umeh, O. (2011). Does the Growth of Structure Affect Our Dynamical Models of the Universe? the Averaging, Backreaction, and Fitting Problems in Cosmology. Rep. Prog. Phys. 74, 112901. arXiv: 1109.2314 [astro-ph.CO]. doi:10.1088/0034-4885/74/11/112901

Clifton, T. (2011). Cosmology without Averaging. Class. Quan. Grav. Class. Quant. Grav., 28, 164011, 2011. arXiv: 1005.0788 [gr-qc]. doi:10.1088/0264-9381/28/16/164011

Chataignier, L., and Krämer, M. (2021). Unitarity of Quantum-Gravitational Corrections to Primordial Fluctuations in the Born-Oppenheimer Approach. Phys. Rev. D 103 (6), 066005. arXiv: 2011. 06426 [gr-qc]. doi:10.1103/physrevd.103.066005

Clarkson, C., Ananda, K., and Larena, J. (2009). The Influence of Structure Formation on the Cosmic Expansion. Phys. Rev. D 80, 083525. arXiv: 0907.3377 [astro-ph.CO]. doi:10.1103/physrevd.80.083525

Clarkson, C., Ellis, G., Larena, J., and Umeh, O. (2011). Does the Growth of Structure Affect Our Dynamical Models of the Universe? The Averaging, Backreaction, and Fitting Problems in Cosmology. Rep. Prog. Phys. 74, 112901. arXiv: 1109.2314 [astro-ph.CO]. doi:10.1088/0034-4885/74/11/112901

Clifton, T., and Ferreira, P. G. (2009). Archipelagian Cosmology: Dynamics and Observables in a Universe with Discretized Matter Content. Erratum: Phvs.Rev.D 84, 109902 (2011)]. Phys. Rev. D 80, 103503. [arXiv: 0907.4109 [astro-ph.CO]. doi:10.1103/physrevd.80.103503

Cole, S., Percival, W. J., Peacock, J. A., Norberg, P., Baugh, C. M., Frenk, C. S., et al. (2005). The 2dF Galaxy Redshift Survey: Power-Spectrum Analysis of the Final
Data Set and Cosmological Implications. Mon. Not. Roy. Astron. Soc. 362, 505-534. arXiv: astro- ph/0501174. doi:10.1111/j.1365-2966.2005.09318.x

Colless, M., Dalton, G. B., Maddox, S. J., Sutherland, W. J., Norberg, P., Cole, S., et al. (2001). The 2dF Galaxy Redshift Survey: Spectra and Redshifts. Mon. Not. Roy. Astron. Soc. 328, 1039. arXiv: astro-ph/0106498. doi:10.1046/j.13658711.2001.04902.x

Dappiaggi, C., Fredenhagen, K., and Pinamonti, N. (2008). Stable Cosmological Models Driven by a Free Quantum Scalar Field. Phys. Rev. D 77, 104015. arXiv: 0801.2850 [gr-qc]. doi:10.1103/physrevd.77.104015

Dappiaggi, C., Hack, T.-P., Möller, J., and Pinamonti, N. (2010). Dark Energy from Quantum Matter. Available at: arXiv: 1007. 5009 [astro-ph.CO] (Accessed July $28,2010)$.

Deruelle, N., and Uzan, J.-P. (2018). Relativity in Modern Physics. Oxford: Oxford University Press.

DeWitt, B. S. (1967). Quantum Theory of Gravity. I. The Canonical Theory. Phys. Rev. 160, 1113-1148. doi:10.1103/physrev.160.1113

Di Valentino, E., Anchordoqui, L. A., Akarsu, O., Ali-Haimoud, Y., Amendola, L. Arendse, N., et al. (2020a). Cosmology Intertwined II: The Hubble Constant Tension. Available at: arXiv: 2008.11284 [astro-ph.CO] (Accessed August 25, 2020).

Di Valentino, E., Anchordoqui, L. A., Akarsu, O., Ali-Haimoud, Y., Amendola, L., Arendse, N., et al. (2020b). Cosmology Intertwined III: $\mathrm{F}_{8}$ and $\mathrm{S}_{8}$. Available at: arXiv: 2008.11285 [astro-ph.CO] (Accessed August 25, 2020).

Dimassi, M., and Sjöstrand, J. (1999). Spectral Asymptotics in the Semi-Classical Limit. Cambridge: Cambridge University Press.

Dodelson, S., and Schmidt, F. (2021). Modern Cosmology. Second Edition. Amsterdam: Elsevier - Academic Press.

Dubin, D. A., Hennings, M. A., and Smith, T. B. (1980). Mathematical Aspects of Weyl Quantization and Phase, Vol. 1. Singapore: World Scientific Publishing.

Durrer, R. (2004). "2 Cosmological Perturbation Theory," in Lecture Notes in Physics 653. Editor E. Pa- pantonopoulos, 31-69. arXiv: astro-ph/0402129. doi:10.1007/978-3-540-31535-3_2

Dymnikova, I., and Khlopov, M. (2001). "Decay of Cosmological Constant in SelfConsistent Inflation," Eur. Phys. J. C Decay of Cosmological Constant in Selfconsistent Inflation (Eur. Phys. J. C), 20, 139-146. doi:10.1007/s100520100625

Ellis, G. F. R. (2011). Inhomogeneity Effects in Cosmology. Class. Quan. Grav., 28, , 2011 164001. arXiv: 1103.2335 [astro-ph.CO]. doi:10.1088/0264-9381/28/16/ 164001

Ellis, G. F. R., and Stoeger, W. (1987). The 'fitting Problem' in Cosmology. Class. Quan. Grav., 4, 1697-1729. doi:10.1088/0264-9381/4/6/025

Elizaga Navascués, B., Martín-Benito, M., and Mena Marugán, G. A. (2016). Hybrid Models in Loop Quantum Cosmology. Int. J. Mod. Phys. D 25, 1642007, 2016. arXiv: 1608.05947 [gr-qc]. doi:10.1142/s0218271816420074

Eltzner, B., and Gottschalk, H. (2011). Dynamical Backreaction in RobertsonWalker Spacetime. Rev. Math. Phys. 23, 531-551. arXiv: 1003.3630 [math-ph]. doi:10.1142/s0129055x11004357

J. Erdmenger (Editor) (2009). String Cosmology: Modern String Theory Concepts from the Big Bang to Cosmic Structure. Hoboken: Wiley.

Fanizza, G., Gasperini, M., Marozzi, G., and Veneziano, G. (2020). Generalized Covariant Prescriptions for Averaging Cosmological Observables. J. Cosmol. Astropart. Phys. JCAP 02, 2020, 017. arXiv: 1911.09469 [gr-qc]. doi:10.1088/ $1475-7516 / 2020 / 02 / 017$

Fernández-Méndez, M., Mena Marugán, G. A., Olmedo, J., and Velhinho, J. M. (2012). Unique Fock Quantization of Scalar Cosmological Perturbations. Phys. Rev. D 85, 103525. arXiv: 1203.2525 [gr-qc]. doi:10.1103/ physrevd.85.103525

Finelli, F., Marozzi, G., Starobinsky, A. A., Vacca, G. P., and Venturi, G. (2009). Generation of Fluctuations during Inflation: Comparison of Stochastic and Field-Theoretic Approaches. Phys. Rev. D 79, 044007. arXiv: 0808.1786 [hepth]. doi:10.1103/physrevd.79.044007

Finelli, F., Marozzi, G., Vacca, G. P., and Venturi, G. (2002). Energy-momentum Tensor of Field Fluctuations in Massive Chaotic Inflation. Phys. Rev. D 65, 103521. arXiv: gr-qc/0111035. doi:10.1103/physrevd.65.103521

Finelli, F., Marozzi, G., Vacca, G. P., and Venturi, G. (2011). Backreaction during Inflation: A Physical Gauge Invariant Formulation. Phys. Rev. Lett. 106, 121304. arXiv: 1101.1051 [gr-qc]. doi:10.1103/ PhysRevLett.106.121304 
Finelli, F., Marozzi, G., Vacca, G. P., and Venturi, G. (2004). Energy-momentum Tensor of Cosmological Fluctuations during Inflation. Phys. Rev. D 69, 123508. arXiv: gr-qc/0310086. doi:10.1103/PhysRevD.69.123508

Fischetti, M. V., Hartle, J. B., and Hu, B. L. (1979). Quantum Effects in the Early Universe. I. Influence of Trace Anomalies on Homogeneous, Isotropic, Classical Geometries. Phys. Rev. D 20, 1757-1771. doi:10.1103/ physrevd.20.1757

Flanagan, É. É. (2005). Can Superhorizon Perturbations Drive the Acceleration of the Universe? Phys. Rev. D 71, 103521. arXiv: hep-th/0503202. doi:10.1103/ physrevd.71.103521

Flanagan, E. E., and Wald, R. M. (1996). Does Back Reaction Enforce the Averaged Null Energy Condition in Semiclassical Gravity? Phys. Rev. D 54, 6233-6283. arXiv: gr-qc/9602052. doi:10.1103/physrevd.54.6233

Folland, G. B. (1989). Harmonic Analysis in Phase Space. Princeton: Princeton University Press.

Ford, L. H. (2005). "Spacetime in Semiclassical Gravity," in 100 Years of Relativity: Space-Time Structure: Einstein and beyond. Editor A. Ashtekar, pp. 293-310. arXiv: gr-qc/0504096. doi:10.1142/9789812700988_0011

Friedman, A. (1922). Uber die Krümmung des Raumes. Z. Physik 10, 377-386. doi: $10.1007 / \mathrm{bf} 01332580$

Friedmann, A. (1924). Uber die Möglichkeit einer Welt mit konstanter negativer Krümmung des Raumes. Z. Physik 21, 326-332. doi:10.1007/bf01328280

Fulling, S. A., Parker, L., and Hu, B. L. (1974). Conformal Energy-Momentum Tensor in Curved Spacetime: Adiabatic Regularization and Renormalization. Phys. Rev. D 10, 3905-3924. doi:10.1103/physrevd.10.3905

Fulling, S. A., and Parker, L. (1974). Renormalization in the Theory of a Quantized Scalar Field Interacting with a Robertson-walker Spacetime. Ann. Phys. 87, 176-204. doi:10.1016/0003-4916(74)90451-5

Fulling, S. A. (1989). London Mathematical Society Student Texts. Cambridge: Cambridge University Press, 7.

Gamov, G. (1948a). The Evolution of the Universe. Nature 162, 680-682.

Gamov, G. (1948b). The Origin of Elements and the Separation of Galaxies. Phys. Rev. 74, 505.

Gasperini, M., Marozzi, G., Nugier, F., and Veneziano, G. (2011). Light-cone Averaging in Cosmology: Formalism and Applications. J. Cosmol. Astropart. Phys. JCAP 07, 2011, 008. arXiv: 1104.1167 [astro-ph.CO]. doi:10.1088/14757516/2011/07/008

Geshnizjani, G., and Brandenberger, R. (2002). Back Reaction and the Local Cosmological Expansion Rate. Phys. Rev. D 66, 123507. arXiv: gr-qc/ 0204074. doi:10.1103/PhysRevD.66.123507

Geshnizjani, G., and Brandenberger, R. (2005). Back Reaction of Perturbations in Two Scalar Field Inflationary Models JCAP 04, 006. arXiv: hep-th/0310265. doi:10.1088/1475-7516/2005/04/006

Giesel, K., Tambornino, J., and Thiemann, T. (2009). Born-Oppenheimer Decomposition for Quantum fields on Quantum Spacetimes. Available at: arXiv: 0911.5331 [gr-qc] (Accessed November 27, 2009).

Gottschalk, H., and Siemssen, D. (2018). The Cosmological Semiclassical Einstein Equation as an Infinite-Dimensional Dynamical System. Available at: arXiv: 1809.03812 [math-ph] (Accessed September 11, 2018).

Green, S. R., and Wald, R. M. (2011). A New Framework for Analyzing the Effects of Small Scale Inhomogeneities in Cosmology. Phys. Rev. D 83, 084020. arXiv: 1011.4920 [gr-qc]. doi:10.1103/physrevd.83.084020

Green, S. R., and Wald, R. M. (2013). Examples of Backreaction of Small-Scale Inhomogeneities in Cosmology. Phys. Rev. D 87, 124037. arXiv: 1304.2318 [gr-qc]. doi:10.1103/physrevd.87.124037

Green, S. R., and Wald, R. M. (2014). How Well Is Our Universe Described by an FLRW Model? Class. Quan. Grav. 31, 234003. arXiv:1407.8084 [gr-qc]. doi:10.1088/0264-9381/31/23/234003

Green, S. R., and Wald, R. M. (2012). Newtonian and Relativistic Cosmologies. Phys. Rev. D 85, 063512. arXiv: 1111.2997 [gr-qc]. doi:10.1103/ physrevd.85.063512

Grishchuk, L. P. (1977). Graviton Creation in the Early Universe. Ann. NY Acad. Sci. 302, 439-444. doi:10.1111/j.1749-6632.1977.tb37064.x

Haag, R. (1992). Local Quantum Physics: Fields, Particles, Algebras. Berlin: Springer-Verlag.

Hack, T.-P. ( 2013). The Lambda CDM Model in Quantum Field Theory on Curved Spacetime and Dark: Radiation. Available at: arXiv: 1306.3074 [gr-qc] (Accessed June 13, 2013).
Halliwell, J. J., and Hawking, S. W. (1987). "The Origin of Structure in the Universe," in Adv. Ser. Astrophys. Cosmol. 3. Editors L. Z. Fang and R. Ruffini, pp. 277-291.

Halliwell, J. J., and Hawking, S. W. (1985). "Origin of Structure in the Universe. Phys. Rev. D" in The Origin of Structure in the Universe. Li-Zhi Fang and R. Ruffini Editors, 31, 1777-1791. doi:10.1103/PhysRevD.31.1777

Heinesen, A. (2021a). Multipole Decomposition of Redshift Drift - Model Independent Mapping of the Expansion History of the Universe. Phys. Rev. $D$ 103.2, 023537. arXiv: 2011.10048 [gr-qc]. doi:10.1103/ physrevd.103.023537

Heinesen, A. (2021b). Redshift Drift as a Model Independent Probe of Dark Energy. Phys. Rev. D 103, L081302. arXiv: 2102.03774 [gr-qc]. doi:10.1103/ PhysRevD.103.L081302

Heinesen, A., and Buchert, T. (2020). Solving the Curvature and Hubble Parameter Inconsistencies through Structure Formation-Induced Curvature. Class. Quan. Grav. 37 (16), 164001, 2020. arXiv: 2002.10831 [gr-qc]. doi:10.1088/1361-6382/ ab954b

Heymans, C., Tröster, T., Asgari, M., Blake, C., Hildebrandt, H., Joachimi, B., et al. (2021). KiDS-1000 Cosmology: Multi-Probe Weak Gravitational Lensing and Spectroscopic Galaxy Clustering Constraints. Astron. Astrophys. 646, A140, 2021. arXiv: 2007.15632 [astro-ph.CO]. doi:10.1051/ 0004-6361/202039063

Hollands, S., and Wald, R. M. (2001). Local Wick Polynomials and Time Ordered Products of Quantum Fields in Curved Spacetime. Commun. Math. Phys. 223, 289-326. arXiv: gr-qc/0103074. doi:10.1007/s002200100540

Hörmander, L. (1985a). The Analysis of Linear Partial Differential Operators II: Differential Operators with Constant Coefficients. Berlin, Heidelberg: SpringerVerlag.

Hörmander, L. (1985b). The Analysis of Linear Partial Differential Operators III: Pseudo Differential Operators. Berlin, Heidelberg: Springer-Verlag.

Hu, B. L., and Matacz, A. (1995). Back Reaction in Semiclassical Gravity: The Einstein-Langevin Equation. Phys. Rev. D 51, 1577-1586. arXiv: gr-qc/9403043. doi:10.1103/physrevd.51.1577

Hu, B. L., and Parker, L. (1978). Anisotropy Damping through Quantum Effects in the Early Universe. Phys. Rev. D 17, 933-945. [Erratum: Phys.Rev.D 17, 3292 (1978)]. doi:10.1103/physrevd.17.933

Hu, B. L., and Parker, L. E. (1977). Effect of Gravitation Creation in Isotropically Expanding Universes. Phys. Let. A. 63 (3), 214-220. doi:10.1016/0375-9601(77) 90880-5

Hu, B. L., and Verdaguer, E. (2008). Stochastic Gravity: Theory and Applications. Living Rev. Relativ. 11, 3. 2008. arXiv: 0802.0658 [gr-qc]. doi:10.12942/lrr2008-3

Hu, B. L., and Verdaguer, E. (2020). "Semiclassical and Stochastic Gravity: Quantum, Field Effects on Curved Spacetime," in Cambridge Monographs on Mathematical Physics (Cambridge: Cambridge University Press).

Jordan, R. D. (1987). Stability of Flat Spacetime in Quantum Gravity. Phys. Rev. D 36, 3593-3603. doi:10.1103/physrevd.36.3593

Jordan, R. (1986). Effective Field Equations for Expectation Values. Phys. Rev. D 33, 444454. doi:10.1103/physrevd.33.444

Kantowski, R. (1969). Corrections in the Luminosity-Redshift Relations of the Homogeneous Fried-Mann Models. ApJ 155, 89. doi:10.1086/149851

Kiefer, C. (1987). Continuous Measurement of Mini-Superspace Variables by Higher Multipoles. Class. Quan. Grav. 4, 1369-1382. doi:10.1088/0264-9381/4/ $5 / 031$

Kiefer, C., and Singh, T. P. (1991). Quantum Gravitational Corrections to the Functional Schrödinger Equation. Phys. Rev. D 44, 1067-1076. doi:10.1103/ physrevd.44.1067

Kiefer, C. (2007). Quantum Gravity. Second Edition. Oxford: Oxford University Press.

Kiefer, C. (1994). "The Semiclassical Approximation to Quantum Gravity," in Lect. Notes Phys. 434. Editors J. Ehlers and H. Friedrich, pp. 170-212. arXiv: gr-qc/9312015.

Kiefer, C. (1994). " The Semiclassical Approximation to Quantum Gravity". In: Lect. Notes Phys. 434. Ed. by H. J. Ehlers Friedrich, pp. 170-212. arXiv: gr- qc/ 9312015. doi:10.1007/3-540-58339-4_19

Kolb, E. W., Marra, V., and Matarrese, S. (2010). Cosmological Background Solutions and Cosmological Backreactions. Gen. Relativ Gravit. 42, 1399-1412. arXiv: 0901.4566 [astro-ph.CO]. doi:10.1007/s10714-0090913-8 
Kolb, E. W., Matarrese, S., Notari, A., and Riotto, A. (2005). The Effect of Inhomogeneities on the Expansion Rate of the Universe. Phys. Rev. D 71, 023524. arXiv: hep-ph/0409038. doi:10.1103/physrevd.71.023524

Koksbang, S. M. (2019). Another Look at Redshift Drift and the Backreaction Conjecture. J. Cosmol. Astropart. Phys. JCAP 10, 2019, 036. arXiv: 1909.13489 [astro-ph.CO]. doi:10.1088/1475-7516/2019/10/036

Koksbang, S. M. (2020). Observations in Statistically Homogeneous, Locally Inhomogeneous Cosmological Toy Models without FLRW Backgrounds. Mon. Not. Roy. Astron. Soc. 498, L135-L139. arXiv: 2008.07108 [astroph.CO]. doi:10.1093/mnrasl/slaa14610.1093/mnrasl/slaa146

Koksbang, S. M. (2021). Searching for Signals of Inhomogeneity Using Multiple Probes of the Cosmic Expansion Rate H(z). Phys. Rev. Lett. 126, 231101. arXiv: 2105. 11880 [astro-ph.CO]. doi:10.1103/PhysRevLett.126.231101

Krasinski, A., and Bolejko, K. (2011). Redshift Propagation Equations in the $\beta^{\prime}=0$ Szekeres Models. Phys. Rev. D 83, 083503. arXiv: 1007.2083 [gr-qc].

Kristian, J., and Sachs, R. K. (1966). Observations in Cosmology. ApJ 143, 379-399. doi:10.1086/148522

Lemaître, A. G. (1931). A Homogeneous Universe of Constant Mass and Increasing Radius Accounting for the Radial Velocity of Extra-galactic Nebulae. Monthly Notices R. Astronomical Soc. 91 (5), 483-490.

Li, N., and Schwarz, D. (2008). Scale Dependence of Cosmological Backreaction. Phys. Rev. D 78, 083531. arXiv: 0710.5073 [astro-ph]. doi:10.1103/ physrevd.78.083531

Lindquist, R. W., and Wheeler, J. A. (1957). Dynamics of a Lattice Universe by the Schwarzschild-Cell Method. Rev. Mod. Phys. 29 (3), 432-443. doi:10.1103/ revmodphys.29.432

Löffler, F., Faber, J., Bentivegna, E., Bode, T., Diener, P., Haas, R., et al. (2012). The Einstein Toolkit: A Community Computational Infrastructure for Relativistic Astrophysics. Class. Quan. Grav. 29, 115001. arXiv: 1111.3344 [gr-qc]. doi:10.1088/0264-9381/29/11/115001

Lombardo, F., and Mazzitelli, F. D. (1996). Coarse Graining and Decoherence in Quantum Field Theory. Phys. Rev. D 53, 2001-2011. arXiv: hep-th/9508052. doi:10.1103/physrevd.53.2001

Losic, B., and Unruh, W. G. (2008). Cosmological Perturbation Theory in SlowRoll Spacetimes. Phys. Rev. Lett. 101, 111101. arXiv: 0804.4296 [gr-qc]. doi:10.1103/PhysRevLett.101.111101

Losic, B., and Unruh, W. G. (2005). Long-wavelength Metric Backreactions in SlowRoll Inflation. Phys. Rev. D 72, 123510. arXiv: gr-qc/0510078. doi:10.1103/ PhysRevD.72.123510

Marozzi, G., Vacca, G. P., and Brandenberger, a. R. H. (2013). Cosmological Backreaction for a Test Field Observer in a Chaotic Inflationary Model. J. Cosmol. Astropart. Phys. JCAP 02, 2013, 027. arXiv: 1212.6029 [hep-th]. doi:10.1088/1475-7516/2013/02/027

Matsui, H., and Watamura, N. (2020). Quantum Spacetime Instability and Breakdown of Semiclassical Gravity. Phys. Rev. D 101.2, 025014. arXiv: 1910. 02186 [gr-qc]. doi:10.1103/physrevd.101.025014

Macpherson, H., Price, D., and Laskv, P. (2019). Einstein's Universe: Cosmological Structure Formation in Numerical Relativity. Phys. Rev. D 99 (6), 063522. arXiv: 1807.01711 [astro-ph.CO].

Manzano, D. (2020). A Short Introduction to the Lindblad Master Equation. AIP Adv. 10, 025106. doi:10.1063/1.5115323

Marra, V., Kolb, E. W., and Matarrese, S. (2008). Light-cone Averages in a SwissCheese Universe. Phys. Rev. D 77, 023003. arXiv: 0710.5505 [astro-ph]. doi:10.1103/physrevd.77.023003

Martínez, F. B., and Olmedo, J. (2016). Primordial Tensor Modes of the Early Universe. Phys. Rev. D 93, 124008. arXiv:1605.04293 [gr-qc]. doi:10.1103/ physrevd.93.124008

Matsui, H., and Watamura, N. (2020). Quantum, Spacetime Instability and Breakdown of Semiclassical Gravity. Phys. Rev. D 101 (2), 025014. arXiv: 1910.02186 [gr-qc]. doi:10.1103/physrevd.101.025014

Meda, P., Pinamonti, N., and Siemssen, D. (2020). Existence and Uniqueness of Solutions of the Semiclassical, Einstein Equation in Cosmological Models. Available at: arXiv: 2007.14665 [math-ph] (Accessed July 29, 2020).

Mertens, J. B., Giblin, J. T., and Starkman, G. D. (2016). Integration of Inhomogeneous Cosmological Spacetimes in the BSSN Formalism. Phys. Rev. D 93, 124059. arXiv: 1511.01106 [gr-qc]. doi:10.1103/physrevd.93.124059

Mukhanov, V. F. (2005). Physical Foundations of Cosmology. Cambridge: Cambridge University Press.
Mukhanov, V. F. (1988). Quantum, Theory of Gauge Invariant Cosmological Perturbations. Sov. Phys. JETP 67, 1297-1302.

Mukhanov, V. F., Abramo, L. R. W., and Brandenberger, R. H. (1997). Backreaction Problem for Cosmological Perturbations. Phys. Rev. Lett. 78, 1624-1627. arXiv: gr-qc/9609026. doi:10.1103/physrevlett.78.1624

Neuser, J., Schander, S., and Thiemann, T. (2019). Quantum Cosmological Backreactions II: Purely Homogeneous Quantum Cosmology. Available at: arXiv: 1906.08185 [gr-qc] (Accessed June 19, 2019).

Panati, G., Spohn, H., and Teufel, S. (2003). Space-Adiabatic Perturbation Theory. Adv. Theor. Math. Phys. 7, 145-204. arXiv: math-ph/0201055. doi:10.4310/ atmp.2003.v7.n1.a6

Paranjape, A. (2012). "Averaging the Inhomogeneous Universe," in Vignettes in Gravitation and Cosmology, 77-113. doi:10.1142/ 9789814322072_0004

Paranjape, A., and Singh, T. P. (2008). Cosmic Inhomogeneities and Averaged Cosmological Dynamics. Phys. Rev. Lett. 101, 181101. 2008. arXiv: 0806.3497 [astro-ph]. doi:10.1103/physrevlett.101.181101

Paranjape, A., and Singh, T. P. (2007). The Spatial Averaging Limit of Covariant Macroscopic Gravity: Scalar Corrections to the Cosmological Equations. Phys. Rev. D 76, 044006, 2007. arXiv: gr-qc/0703106. doi:10.1103/ physrevd.76.044006

Parker, L. E., and Raval, A. (1999). Nonperturbative Effects of Vacuum Energy on the Recent Expansion of the Universe, [Erratum: Phvs. Rev. D 67, 029901 (2003)]. Phys. Rev. D 60, 063512. arXiv: gr-qc/9905031. doi:10.1103/ physrevd.60.063512

Parker, L., and Fulling, S. A. (1974). Adiabatic Regularization of the EnergyMomentum Tensor of a Quantized Field in Homogeneous Spaces. Phys. Rev. D 9, 341-354. doi:10.1103/physrevd.9.341

Pattison, C., Vennin, V., Assadullahi, H., and Wands, D. (2019). Stochastic Inflation beyond Slow Roll. J. Cosmol. Astropart. Phys. JCAP 07, 2019, 031. arXiv: 1905.06300 [astro-ph.CO]. doi:10.1088/1475-7516/2019/07/031

Paz, J. P., and Sinha, S. (1992). Decoherence and Back Reaction in Quantum Cosmology: Multidimensional Minisuperspace Examples. Phys. Rev. D 45, 2823-2842. doi:10.1103/physrevd.45.2823

Paz, J. P., and Sinha, S. (1991). Decoherence and Back Reaction: The Origin of the Semiclassical Einstein Equations. Phys. Rev. D 44, 1038-1049. doi:10.1103/ physrevd.44.1038

Peebles, P. J. E. (1982). Large Sclae Background Temperature and Mass Fluctuations Due to Scale- Invariant Primeval Perturbations. Astrophys. J. 263, L1. doi:10.1086/183911

Peebles, P. J. E., Alimi, J.-M., and Fuözfa, A. (2010). Phenomenology of the Invisible Universe. AIP Conf. Proc. 1241, 175182. arXiv: 0910.5142 [astroph.CO]. doi:10.1063/1.3462631

Peebles, P. J. E. (2010). "Phenomenology of the Invisible Universe," in AIP Conference Proceedings. 1241, 175-182. arXiv: 0910.5142 [astro-ph.CO].

Peebles, P. J. E., and Ratra, B. (2003). "The Cosmological Constant and Dark Energy," in Rev. Mod. Phys. 75. Editor J-P. Hsu and D. Fine, pp. 559-606. arXiv: astro-ph/0207347. doi:10.1103/revmodphys.75.559

Penzias, A. A., and Wilson, R. W. (1965). A Measurement of Excess Antenna Temperature at $4080 \mathrm{Mc} / \mathrm{s}$. ApJ 142, 419-421. doi:10.1086/148307

Perlmutter, S., Aldering, G., Goldhaber, G., Knop, R. A., Nugent, P., Castro, P. G., et al. (1999). Measurements of $\Omega$ and $\Lambda$ from 42 High-Redshift Supernovae. ApJ 517, 565-586. arXiv: astro-ph/9812133. doi:10.1086/307221

Pesce, D. W. T., Braatz, J. A., Reid, M. J., Riess, A. G., Scolnic, D., Condon, J. J., et al. (2020). The Megamaser Cosmology Project. XIII. Combined Hubble Constant Constraints. Astrophys. J. Lett. 891 (1), L1. arXiv: 2001.09213 [astro-ph.CO]. doi:10.3847/2041-8213/ab75f0

Pinamonti, N. (2011). On the Initial Conditions and Solutions of the Semiclassical Einstein Equations in a Cosmological Scenario. Commun. Math. Phys. 305, 563-604. arXiv: 1001.0864 [gr-qc]. doi: $10.1007 / \mathrm{s} 00220-011-1268-\mathrm{z}$

Pinamonti, N., and Siemssen, D. (2015). Global Existence of Solutions of the Semiclassical Einstein Equation for Cosmological Spacetimes. Commun. Math. Phys. 334 (1), 171-191. arXiv: 1309. 6303 [math-ph]. doi:10.1007/s00220-0142099-5

Radzikowski, 入. I. J. (2008). Stable, Renormalizable, Scalar Tachyonic Quantum, Field Theory with, Chronology Protection. Available at: arXiv: 0804.4534 [math-ph] (Accessed April 29, 2008). 
Ramsey, S. A., and Hu, B. L. (1997). Nonequilibrium Inflaton Dynamics and Reheating: Back Reaction of Parametric Particle Creation and Curved Spacetime Effects, [Erratum: Phys. Rev. D 57, 3798 (1998)]. Phys. Rev. D 56, 678-705. arXiv: hep-ph/9706207. doi:10.1103/physrevd.56.678

Riess, A. G., Casertano, S., Yuan, W., Macri, L. M., and Scolnic, D. (2019). Large Magellanic Cloud Cepheid Standards Provide a 1\% Foundation for the Determination of the Hubble Constant and Stronger Evidence for Physics beyond $\Lambda$ CDM. Astrophys. J. 876 (1), 85, 2019. arXiv: 1903.07603 [astro-ph.CO].

Riess, A. G., Filippenko, A. V., Challis, P., Clocchiatti, A., Diercks, A., Garnavich, P. M., et al. (1998). Observational Evidence from Supernovae for an Accelerating Universe and a Cosmological Constant. Astron. J. 116, 1009-1038. arXiv: astroph/9805201. doi:10.1086/300499

Robertson, H. P. (1933). Relativistic Cosmology. Rev. Mod. Phys. 5, 62-90. doi:10.1103/revmodphys.5.62

Räsänen, S. (2009). Light Propagation in Statistically Homogeneous and Isotropic Dust Universes. J. Cosmol. Astropart. Phys. JCAP 02, 2009, 011. arXiv: 0812.2872 [astro-ph]. doi:10.1088/1475-7516/2009/02/011

Räsänen, S. (2010). Light Propagation in Statistically Homogeneous and Isotropic Universes with General Matter Content. J. Cosmol. Astropart. Phys. JCAP 03, 2010, 018. arXiv: 0912.3370 [astro-ph.CO]. doi:10.1088/1475-7516/2010/ $03 / 018$

Ross, A. J., Bautista, J., Tojeiro, R., Alam, S., Bailey, S., Burtin, E., et al. (2020). The Completed SDSS-IV Extended Baryon Oscillation Spectroscopic Survey: LargeScale Structure Catalogues for Cosmological Analysis. Mon. Not. Roy. Astron. Soc. 498 (2), 2354-2371. arXiv: 2007.09000 [astro-ph.CO]. doi:10.1093/mnras/ staa2416

Rovelli, C., and Vidotto, F. (2008). Stepping Out of Homogeneity in Loop Quantum Cosmology. Class. Quan. Grav. 25, 225024. arXiv: 0805.4585 [grqc]. doi:10.1088/0264-9381/25/22/225024

Rovelli, C. (2010). "Quantum Gravity," in Cambridge Monographs on Mathematical Physics. Cambridge: Cambridge University Press.

Schander, S., and Thiemann, T. (2019a). Quantum Cosmological Backreactions I: Cosmological Space Adiabatic Perturbation Theory. Available at: arXiv: 1906. 08166 [gr-qc] (Accessed June 19, 2019).

Schander, S., and Thiemann, T. (2019b). Quantum, Cosmological Backreactions III: Deparametrized Quantum, Cosmological Perturbation Theory. Available at: arXiv: 1906.08194 [gr-qc] (Accessed June 19, 2019).

Schander, S., and Thiemann, T. (2019c). Quantum, Cosmological Backreactions IV: Constrained Quantum, Cosmological Perturbation Theory. Available at: arXiv: 1909.07271 [gr-qc] (Accessed September 16, 2019).

Schlosshauer, M. A. (2007). Decoherence and the Quantum-To-Classical Transition. Berlin, Heidelberg: Springer-Verlag.

Sinha, S., and Hu, B. L. (1991). Validity of the Minisuperspace Approximation: An Example from Interacting Quantum Field Theory. Phys. Rev. D 44, 1028-1037. doi:10.1103/physrevd.44.1028

Spergel, D. N. (2015). The Dark Side of Cosmology: Dark Matter and Dark Energy. Science 347, 1100-1102. doi:10.1126/science.aaa0980

Starobinsky, A. A. (1987). "A New Type of Isotropic Cosmological Models without Singularity," in Quantum Cosmology, Advanced Series in Astrophysics and Cosmology 3. Editors I. M. Khalatnikov and V. P. Mineev, pp. 130-133.

Starobinsky, A. A. (1982). "Dynamics of Phase Transition in the New Inflationary Universe Scenario and Generation of Perturbations". In: Phys. Lett. B 117, 175-178.

Starobinsky, A. A. (1988). "Stochastic de Sitter (inflationary) stage in early Universe". In: Field Theory, Quantum Gravity and Strings. Lecture Notes in Physics. Editors H. J. de Vega and N. Sánchez. Springer, Berlin, Heidelberg.

Starobinsky, A. A., and Yokoyama, J. i. (1994). Equilibrium state of a selfinteracting scalar field in the de Sitter background. Phys. Rev. D 50, 6357-6368. arXiv: astro-ph/9407016. doi:10.1103/physrevd.50.6357

Stottmeister, A. (2015). On the Embedding of Quantum, Field Theory on Curved Spacetimes into Loop Quantum Gravity. PhD thesis. Nürnberg: FriedrichAlexander-Universität Erlangen.

Stottmeister, A., and Thiemann, T. (2016a). Coherent States, Quantum Gravity, and the Born-Oppenheimer Approximation. I. General Considerations. J. Math. Phys. 57 (6), 063509. arXiv: 1504.02169 [math-ph]. doi:10.1063/ 1.4954228

Stottmeister, A., and Thiemann, T. (2016b). Coherent States, Quantum Gravity, and the Born- Oppenheimer Approximation. II. Compact Lie Groups. J. Math. Phys. 57, 073501. arXiv: 1504.02170 [math-ph]. doi:10.1063/1.49548037
Stottmeister, A., and Thiemann, T. (2016c). Coherent States, Quantum Gravity, and the Born-Oppenheimer Approximation. III.: Applications to Loop Quantum Gravity. J. Math. Phys. 57, 083509. arXiv: 1504.02171 [math-ph]. doi:10.1063/1.4960823

Sussman, R. A. (2011). Back-reaction and Effective Acceleration in Generic LTB Dust Models. Class. Quan. Grav. 28, 235002. arXiv: 1102.2663 [gr-qc]. doi:10.1088/0264-9381/28/23/235002

Teufel, S. (2003). "Adiabatic Perturbation Theory in Quantum Dynamics," in Lecture Notes in Mathematics. Berlin: Springer-Verlag, 1821.

Thiemann, T. (2008). Modern Canonical Quantum General Relativity. Cambridge: Cambridge University Press.

Tomita, K. (2000). Distances and Lensing in Cosmological Void Models. Astrophys. J. 529, 38. arXiv: astro-ph/9906027.

Tsamis, N. C., and Woodard, R. P. (1993). Relaxing the Cosmological Constant. Phys. Lett. B 301, 351-357. doi:10.1016/0370-2693(93)91162-G

Tsamis, N. C., and Woodard, R. P. (1996). Quantum Gravity Slows Inflation. Nucl. Phys. B 474, 235-248. arXiv: hep-ph/9602315. doi:10.1016/0550-3213(96) 00246-5

Tsamis, N. C., and Woodard, R. P. (2005). Stochastic Quantum Gravitational Inflation. Nucl. Phys. B 724, 295-328. arXiv: gr-qc/0505115. doi:10.1016/ j.nuclphysb.2005.06.031

Unruh, W. G. (1998). " Cosmological Long Wavelength Perturbations". In: arXiv: astro-ph/9802323.

Vidotto, F. (2011). Spinfoam Cosmology. J. Phys. Conf. Ser. 314, 012049, 2011. arXiv: 1011.4705 [gr-qc]. doi:10.1088/1742-6596/314/1/012049

Vilenkin, A. (1989). Interpretation of the Wave Function of the Universe. Phys. Rev. D 39, 1116-1122. doi:10.1103/physrevd.39.1116

Wald, R. M. (1977). The Back Reaction Effect in Particle Creation in Curved Spacetime. Commun. Math. Phys. 54, 1-19. doi:10.1007/bf01609833

Wald, R. M. (1978). Trace Anomaly of a Conformally Invariant Quantum Field in Curved Spacetime. Phys. Rev. D 17, 1477-1484. doi:10.1103/ physrevd.17.1477

Walker, A. G. (1937). On Milne's Theory of World-Structure. Proc. Lond. Math. Soc. s2-42 (1), 90-127. doi:10.1112/plms/s2-42.1.90

Wheeler, J. A. (1957). On the Nature of Quantum Geometrodynamics. Ann. Phys. 2, 604-614. doi:10.1016/0003-4916(57)90050-7

Wiltshire, D. L. (2009). Average Observational Quantities in the Timescape Cosmology. Phys. Rev. D 80, 123512. arXiv: 0909.0749 [astro-ph.CO]. doi:10.1103/physrevd.80.123512

Zalaletdinov, R. (1997). Averaging Problem in General Relativity, Macroscopic Gravity and Using Einstein's Equations in Cosm,ol,ogy. Bull. Astron. Soc. India 25, 401-416. arXiv: gr-qc/9703016.

Zalaletdinov, R. (2008). The Averaging Problem in Cosmology and Macroscopic Gravity. Int. J. Mod. Phys. A. 23, 1173-1181. arXiv: 0801.3256 [gr-qc]. doi:10.1142/s0217751x08040032

Zel'dovich, Y., Einasto, J., and Shandarin, S. (1982). Giant Voids in the Universe. Nature 300, 407-413.

Zel'dovich, Y., and Starobinsky, A. A. (1972). Particle Production and Vacuum Polarization in an Anisotropic Gravitational Field. Sov. Phys. JETP 34, 1159-1166.

Conflict of Interest: The authors declare that the research was conducted in the absence of any commercial or financial relationships that could be construed as a potential conflict of interest.

Publisher's Note: All claims expressed in this article are solely those of the authors and do not necessarily represent those of their affiliated organizations, or those of the publisher, the editors and the reviewers. Any product that may be evaluated in this article, or claim that may be made by its manufacturer, is not guaranteed or endorsed by the publisher.

Copyright (c) 2021 Schander and Thiemann. This is an open-access article distributed under the terms of the Creative Commons Attribution License (CC $B Y)$. The use, distribution or reproduction in other forums is permitted, provided the original author(s) and the copyright owner(s) are credited and that the original publication in this journal is cited, in accordance with accepted academic practice. No use, distribution or reproduction is permitted which does not comply with these terms. 